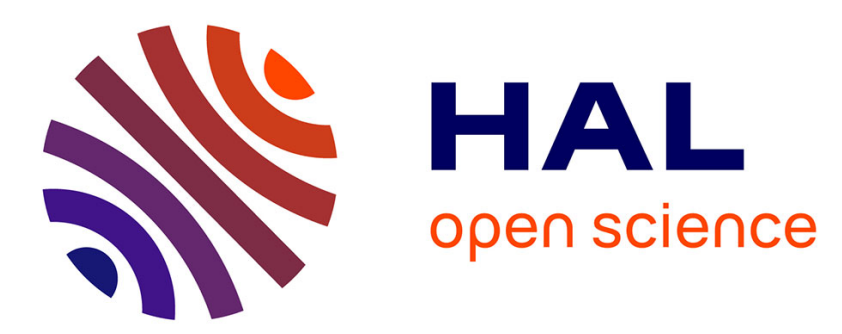

\title{
Lower semicontinuity of integrals of the calculus of variations in Cheeger-Sobolev spaces
}

\author{
Omar Anza Hafsa, Jean Philippe Mandallena
}

\section{To cite this version:}

Omar Anza Hafsa, Jean Philippe Mandallena. Lower semicontinuity of integrals of the calculus of variations in Cheeger-Sobolev spaces. Calc. Var. Partial Differential Equations, 2020, 59 (2). hal02295885

\section{HAL Id: hal-02295885 \\ https://hal.science/hal-02295885}

Submitted on 24 Sep 2019

HAL is a multi-disciplinary open access archive for the deposit and dissemination of scientific research documents, whether they are published or not. The documents may come from teaching and research institutions in France or abroad, or from public or private research centers.
L'archive ouverte pluridisciplinaire $\mathbf{H A L}$, est destinée au dépôt et à la diffusion de documents scientifiques de niveau recherche, publiés ou non, émanant des établissements d'enseignement et de recherche français ou étrangers, des laboratoires publics ou privés. 


\title{
LOWER SEMICONTINUITY OF INTEGRALS OF THE CALCULUS OF VARIATIONS IN CHEEGER-SOBOLEV SPACES
}

\author{
OMAR ANZA HAFSA AND JEAN-PHILIPPE MANDALLENA
}

\begin{abstract}
A necessary condition called $H_{\mu}^{1, p}$-quasiconvexity on $p$-coercive integrands is introduced for the lower semicontinuity with respect to the strong convergence of $L_{\mu}^{p}\left(X ; \mathbb{R}^{m}\right)$ of integral functionals defined on Cheeger-Sobolev spaces. Under polynomial growth conditions it turns out that this condition is necessary and sufficient.
\end{abstract}

\section{Contents}

\begin{tabular}{|c|c|}
\hline & Introduction \\
\hline 2. & The Cheeger-Sobolev spaces \\
\hline 3. & $H_{\mu}^{1, p}$-quasiconvexity is a necessary condition for lower semicontinuity \\
\hline & $H_{\mu}^{1, p}$-quasiconvexity is a necessary and sufficient condition for lower \\
\hline & semicontinuity under polynomial growth \\
\hline 5. & A class of nonconvex integrands without $p$-growth \\
\hline & On the finiteness condition $L(\cdot, \xi) \in L_{\mu}^{1}(X)$ \\
\hline & Proofs of Proposition $|1|$ and Theorem $|4|$ \\
\hline & rences \\
\hline
\end{tabular}

\section{INTRODUCTION}

Let $(X, d, \mu)$ be a metric measure space with $\mu$ a nontrivial locally finite Borel regular measure on $X$ and $(X, d)$ is a separable metric space. Let $p \in] 1, \infty[$. We assume that $\mu$ is doubling and $(X, d, \mu)$ enjoys a $(1, p)$-Poincaré inequality (see \$2), this allows us to define integral functionals of the calculus of variations on Cheeger-Sobolev spaces $H_{\mu}^{1, p}\left(X ; \mathbb{R}^{m}\right)$ on $X$ by

$$
H_{\mu}^{1, p}\left(X ; \mathbb{R}^{m}\right) \ni u \longmapsto I(u)=\int_{X} L\left(x, \nabla_{\mu} u(x)\right) d \mu(x) .
$$

The Cheeger-Sobolev space on $X$ was introduced by Cheeger [Che99] (see $\$ 2$ for the definition). The function $L: X \times \mathbb{M} \longrightarrow[0, \infty]$ is Borel measurable, $\mathbb{M}$ denotes the space of $m$ rows $N$ columns with $N, m \geqslant 1$ integers, and $\nabla_{\mu} u$ is the $\mu$-gradient of $u$.

In this paper, we consider the problem of finding necessary and sufficient conditions on $p$-coercive integrands $L$ (see $(1.3)$ ) for the lower semicontinuity of $I$ with respect to the strong convergence of $L_{\mu}^{p}\left(X ; \mathbb{R}^{m}\right)$ (or equivalently with respect to the sequential weak convergence in $\left.H_{\mu}^{1, p}\left(X ; \mathbb{R}^{m}\right)\right)$. In the setting of Euclidean space $(X, d, \mu)=\left(\Omega,|\cdot-\cdot|,\left.\mathcal{L}_{N}\right|_{\Omega}\right)$ where $\Omega \subset \mathbb{R}^{N}$ is a bounded open subset and $\left.\mathcal{L}_{N}\right\rfloor_{\Omega}$ is the Lebesgue measure on $\Omega$,

Université de Nîmes, Laboratoire MiPA, Site des Carmes, Place Gabriel Péri, 30021 NîMES, FRANCE

E-mail addresses: Omar Anza Hafsa <omar.anza-hafsa@unimes.fr>, Jean-Philippe Mandallena <jean-philippe.mandallena@unimes.fr>.

Key words and phrases. Lower semicontinuity, $H_{\mu}^{1, p}$-quasiconvexity, Integral functionals defined on Cheeger-Sobolev spaces. 
a necessary condition on finite integrands for the weak lower semicontinuity of (1.1) is the quasiconvexity of $L(x, \cdot)$. This condition was introduced by Morrey Mor52 (see also [Dac08, AF84, Mar85]). Later a generalisation for not necessary finite integrand, called $W^{1, p}$-quasiconvexity, was developped by Ball and Murat [BM84] (see also [Man13, Syc15, Kri15]). In the setting of metric measure space we introduce the following condition playing the role of $W^{1, p}$-quasiconvexity, by saying that $L$ is $H_{\mu}^{1, p}$-quasiconvex at $\xi \in \mathbb{M}$ if for $\mu$-a.e. $x \in X$

$$
L(x, \xi) \leqslant \underline{\lim _{\rho \rightarrow 0}} \inf _{\varphi \in H_{\mu, 0}^{1, p}\left(B_{\rho}(x) ; \mathbb{R}^{m}\right)} f_{\bar{B}_{\rho}(x)} L\left(y, \xi+\nabla_{\mu} \varphi(y)\right) d \mu(y) .
$$

The formula on the right hand side of (1.2) already appears in relaxation and homogenization of integral functionals see AHM15, AHM18, AHM17 (see also Man05). In the setting of Euclidean space $(X, d, \mu)=\left(\mathbb{R}^{N},|\cdot-\cdot|, \mu\right)$ with a positive Radon measure compactly supported in $\mathbb{R}^{N}$, necessary conditions for the weak lower semicontinuity was studied in [Fra03, Theorem 4.1, pp. 114]. This was done under Lipschitz condition for $L(x, \cdot)$ and continuity for $L(\cdot, \xi)$, and relies heavily on the use of the Euclidean structure of $\mathbb{R}^{N}$. Here, we establish the necessary condition $(1.2)$ at every $\xi \in \mathbb{M}$ satisfying $L(\cdot, \xi) \in L_{\mu}^{1}(X)$ for Borel measurable integrands which are $p$-coercive, see Theorem 2 . As already said, the $p$-coercivity assumption on the integrand $L$ allows to use in a equivalent way the strong convergence of $L_{\mu}^{p}\left(X ; \mathbb{R}^{m}\right)$ or the sequential weak convergence in $H_{\mu}^{1, p}\left(X ; \mathbb{R}^{m}\right)$, however, the $p$-coercivity condition is essential in our proof; it differs from the Euclidean framework where the quasiconvexity is established as a necessary condition, for the sequential weak convergence in $W^{1, p}\left(\Omega ; \mathbb{R}^{m}\right)$, without requiring any coercivity condition, see [AF84, Theorem [II.2], pp. 134]. The proof uses a Vitali covering of the set where (1.2) does not hold. Then using the $p$-coercivity we are able to construct a sequence of $H_{\mu}^{1, p}\left(X ; \mathbb{R}^{m}\right)$ which strongly converges to 0 in $L_{\mu}^{p}\left(X ; \mathbb{R}^{m}\right)$. The last step consists in using the lower semicontinuity of $I$ and the finitness condition $L(\cdot, \xi) \in L_{\mu}^{1}(X)$ to conclude that the set is necessarily of zero measure. We show that the condition (1.2) turns out to be sufficient (see Theorems 6 and 7 ) when the integrand has $p$-polynomial growth, is lower semicontinuous with respect the second (matrix) variable and that the metric measure space is in addition satisfying the annular decay property and a property of Alexandrov type (or Portmanteau type) for uniformly bounded sequence of nonnegative Borel measures on $X$ (see $\$ 4$ ).

The plan of the paper is as follows. In $\$ 2$ we provide the materials about metric measure spaces and Cheeger-Sobolev spaces we need for our purposes. In $\$ 3$ after introducing the definition and some properties of $H_{\mu}^{1, p}$-quasiconvex integrands, we prove that $H_{\mu}^{1, p}$-quasiconvexity is a necessary condition for the lower semicontinuity with respect to the strong convergence of $L_{\mu}^{p}\left(X ; \mathbb{R}^{m}\right)$. We also show, as an illustration, that convex integrands are $H_{\mu}^{1, p}$-quasiconvex. In $\$ 3.3$ we establish a generalisation of the necessary condition for $p$-coercive and lower semicontinuous abstract functionals. The $\$ 4$ is devoted to the sufficiency of $H_{\mu}^{1, p}$-quasiconvexity for the sequential weak lower semicontinuity of integrals of the calculus of variations, under $p$-polynomial growth. We deduce, in $\$ 4.2$. that $H_{\mu}^{1, p}$-quasiconvexity is a necessary and sufficient condition for the lower semicontinuity with respect to the strong convergence of $L_{\mu}^{p}\left(X ; \mathbb{R}^{m}\right)$ (when $\mu$ is finite see Theorem 6 . and when $\mu$ is not necessarily finite see Theorem 7). In $\$ 5$ we provide and study a class of nonconvex $H_{\mu}^{1, p}$-quasiconvex integrands which may not have polynomial growth, roughly, they are composition of a convex and lower semicontinuous integrand with a finite family of $H_{\mu}^{1, p}$-quasiconvex Carathéodory integrands having $q$-growth with $\left.q \in\right] 1, p[$. In $\$ 6$ we 
discuss the finiteness condition $L(\cdot, \xi) \in L_{\mu}^{1}(X)$ by providing a result (Proposition 5) which gives an indication for the case where $L(\cdot, \xi) \notin L_{\mu}^{1}(X)$, and it is illustrated by Corollary 3 . The last section $\$ 7$ is devoted to the proofs of Proposition 1 and Theorem 4.

\section{Notation.}

- We will denote by $\mathcal{O}(X)$ the set of all open subsets of $(X, d)$, and $\mathcal{B}(X)$ the Borel $\sigma$-algebra of $X$.

- We will denote by $B_{\rho}(x):=\{y \in X: d(x, y)<\rho\}$ the open ball, and by $\bar{B}_{\rho}(x):=\{y \in$ $X: d(x, y) \leqslant \rho\}$ the closed ball, centered at $x$ with radius $\rho>0$.

- For every measurable set $A \subset X$ with positive measure, and for every nonnegative measurable or integrable function $f$ on $A$, we set

$$
f_{A} f d \mu:=\frac{1}{\mu(A)} \int_{A} f(x) d \mu(x) .
$$

- The algebra of Lipschitz functions from $X$ to $\mathbb{R}$ is denoted by $\operatorname{Lip}(X)$.

- We will denote by $L_{\mu, \text { loc* }}^{1}(X)$ the vector space of all measurable functions $u: X \longrightarrow \overline{\mathbb{R}}$ such that

$$
\int_{B}|u| d \mu<\infty \quad \text { for all open ball } B \subset X \text { satisfying } \mu(B)<\infty \text {. }
$$

- We say $u \in L_{\mu, \text { loc }}^{1}(X)$ if for every $x \in X$ there exists $r>0$ such that $\mu\left(B_{r}(x)\right)<\infty$ and $u \in L_{\mu}^{1}\left(B_{r}(x)\right)$. We have $L_{\mu, \text { loc* }}^{1}(X) \subset L_{\mu, \text { loc }}^{1}(X)$ and when $X$ is proper, i.e. the closed balls of $X$ are compact, $L_{\mu, \text { loc* }}^{1}(X)=L_{\mu, \text { loc }}^{1}(X)$.

- Let $L: X \times \mathbb{M} \longrightarrow[0, \infty]$ (resp. $\left.L: X \times \mathbb{R}^{m} \times \mathbb{M} \longrightarrow[0, \infty]\right)$ be a function. We say that $L$ is $p$-coercive if there exists $c>0$ such that for $\mu$-a.e. $x \in X$ it holds

$$
c|\xi|^{p} \leqslant L(x, \xi) \text { for all } \xi \in \mathbb{M}\left(\text { resp. } c|\xi|^{p} \leqslant L(x, v, \xi) \text { for all }(v, \xi) \in \mathbb{R}^{m} \times \mathbb{M}\right) \text {. }
$$

- By $\mathbb{Q}^{m N} \subset \mathbb{M}$ we denote the set of $m$ rows $N$ columns matrices with rational number entries.

\section{The Cheeger-Sobolev spaces}

Let $p>1$ be a real number, let $(X, d, \mu)$ be a metric measure space, where $\mu$ is a nontrivial locally finite Borel regular measure on $X$ and $(X, d)$ is a separable metric space. In what follows, we assume that $\mu$ is doubling, i.e. there exists a constant $C_{d}$ (called doubling constant) such that

$$
\forall x \in X \quad \forall \rho>0 \quad \mu\left(B_{\rho}(x)\right) \leqslant C_{d} \mu\left(B_{\frac{\rho}{2}}(x)\right) .
$$

We begin with the concept of upper gradient introduced by Heinonen and Koskela (see [HK98]).

Definition 1. A Borel function $g: X \longrightarrow[0, \infty]$ is said to be an upper gradient for $f: X \longrightarrow \mathbb{R}$ if $|f(c(1))-f(c(0))| \leqslant \int_{0}^{1} g(c(s)) d s$ for all continuous rectifiable curves $c:[0,1] \longrightarrow X$.

The concept of upper gradient has been generalized by Cheeger as follows (see Che99, Definition 2.8]).

Definition 2. A function $g \in L_{\mu}^{p}(X)$ is said to be a $p$-weak upper gradient for $f \in L_{\mu}^{p}(X)$ if there exist $\left\{f_{n}\right\}_{n} \subset L_{\mu}^{p}(X)$ and $\left\{g_{n}\right\}_{n} \subset L_{\mu}^{p}(X)$ such that for each $n \geqslant 1, g_{n}$ is an upper gradient for $f_{n}, f_{n} \rightarrow f$ in $L_{\mu}^{p}(X)$ and $g_{n} \rightarrow g$ in $L_{\mu}^{p}(X)$. 
The metric measure space $(X, d, \mu)$ enjoys a $(1, p)$-Poincaré inequality with $p \in] 1, \infty[$ if there exist $C_{p}>0$ and $\sigma \geqslant 1$ such that for every $x \in X$ and every $\rho>0$,

$$
f_{B_{\rho}(x)}\left|f(y)-f_{B_{\rho}(x)} f d \mu\right| d \mu(y) \leqslant \rho C_{p}\left(f_{B_{\sigma \rho}(x)} g^{p} d \mu\right)^{\frac{1}{p}}
$$

for every $f \in L_{\mu}^{p}(X)$ and every $p$-weak upper gradient $g \in L_{\mu}^{p}(X)$ for $f$.

From Cheeger and Keith (see [Che99, Theorem 4.38] and [Kei04, Definition 2.1.1 and Theorem 2.3.1]) we have

Theorem 1. If $\mu$ is doubling, i.e. (2.1) holds, and $X$ enjoys a $(1, p)$-Poincaré inequality, i.e. 2.2 holds, then there exist a countable family $\left\{\left(X_{k}, \gamma^{k}\right)\right\}_{k \in \mathbb{N}}$ of $\mu$-measurable disjoint subsets $X_{k}$ of $X$ with $\mu\left(X \backslash \bigcup_{k \in \mathbb{N}} X_{k}\right)=0$ and of functions $\gamma^{k}=\left(\gamma_{1}^{k}, \cdots, \gamma_{N(k)}^{k}\right)$ : $X \longrightarrow \mathbb{R}^{N(k)}$ with $\gamma_{i}^{k} \in \operatorname{Lip}(X)$ satisfying the following properties:

(i) there exists an integer $N \geqslant 1$ such that $N(k) \in\{1, \cdots, N\}$ for all $k \in \mathbb{N}$;

(ii) for every $k \in \mathbb{N}$ and every $f \in \operatorname{Lip}(X)$ there is a unique $D_{\mu}^{k} f \in L_{\mu}^{\infty}\left(X_{k} ; \mathbb{R}^{N(k)}\right)$ such that for $\mu$-a.e. $x \in X_{k}$,

$$
\lim _{\rho \rightarrow 0} \frac{1}{\rho}\left\|f-f_{x}\right\|_{L_{\mu}^{\infty}\left(B_{\rho}(x)\right)}=\lim _{\rho \rightarrow 0} \sup _{y \in B_{\rho}(x)} \frac{\left|f(y)-f(x)+D_{\mu}^{k} f(x) \cdot\left(\gamma^{k}(y)-\gamma^{k}(x)\right)\right|}{\rho}=0,
$$

where $f_{x} \in \operatorname{Lip}(X)$ is given by $f_{x}(y):=f(x)+D_{\mu}^{k} f(x) \cdot\left(\gamma^{k}(y)-\gamma^{k}(x)\right)$; in particular

$$
D_{\mu}^{k} f_{x}(y)=D_{\mu}^{k} f(x) \text { for } \mu \text {-a.a. } y \in X_{k} \text {; }
$$

(iii) the operator $D_{\mu}: \operatorname{Lip}(X) \rightarrow L_{\mu}^{\infty}\left(X ; \mathbb{R}^{N}\right)$ given by

$$
D_{\mu} f:=\sum_{k \in \mathbb{N}} \mathbb{1}_{X_{k}} D_{\mu}^{k} f
$$

where $\mathbb{1}_{X_{k}}$ denotes the characteristic function of $X_{k}$, is linear and, for each $f, g \in$ $\operatorname{Lip}(X)$, one has

$$
D_{\mu}(f g)=f D_{\mu} g+g D_{\mu} f
$$

(iv) for every $f \in \operatorname{Lip}(X), D_{\mu} f=0 \mu$-a.e. on every $\mu$-measurable set where $f$ is constant.

We set $\mathbb{M}=\mathbb{R}^{m \times N}$ where $N$ is given by Theorem $1(\mathrm{i})$, Let $\operatorname{Lip}\left(X ; \mathbb{R}^{m}\right):=[\operatorname{Lip}(X)]^{m}$ and let $\nabla_{\mu}: \operatorname{Lip}\left(X ; \mathbb{R}^{m}\right) \longrightarrow L_{\mu}^{\infty}(X ; \mathbb{M})$ given by

$$
\nabla_{\mu} u:=\left(\begin{array}{c}
D_{\mu} u_{1} \\
\vdots \\
D_{\mu} u_{m}
\end{array}\right) \text { with } u=\left(u_{1}, \cdots, u_{m}\right) .
$$

From Theorem 1 (iii) we see that for every $u \in \operatorname{Lip}\left(X ; \mathbb{R}^{m}\right)$ and every $f \in \operatorname{Lip}(X)$, one has

$$
\nabla_{\mu}(f u)=f \nabla_{\mu} u+D_{\mu} f \otimes u .
$$

Definition 3. The $p$-Cheeger-Sobolev space $H_{\mu}^{1, p}\left(X ; \mathbb{R}^{m}\right)$ is defined as the completion of the space of Lipschitz functions $\operatorname{Lip}\left(X ; \mathbb{R}^{m}\right)$ with respect to the norm

$$
\|u\|_{H_{\mu}^{1, p}\left(X ; \mathbb{R}^{m}\right)}:=\|u\|_{L_{\mu}^{p}\left(X ; \mathbb{R}^{m}\right)}+\left\|\nabla_{\mu} u\right\|_{L_{\mu}^{p}(X ; \mathbb{M})} .
$$


Taking Proposition 1 (ii) below into account, since $\left\|\nabla_{\mu} u\right\|_{L_{\mu}^{p}(X ; \mathrm{M})} \leqslant\|u\|_{H_{\mu}^{1, p}\left(X ; \mathbb{R}^{m}\right)}$ for all $u \in \operatorname{Lip}\left(X ; \mathbb{R}^{m}\right)$ the linear map $\nabla_{\mu}$ from $\operatorname{Lip}\left(X ; \mathbb{R}^{m}\right)$ to $L_{\mu}^{p}(X ; \mathbb{M})$ has a unique extension to $H_{\mu}^{1, p}\left(X ; \mathbb{R}^{m}\right)$ which will still be denoted by $\nabla_{\mu}$ and will be called the $\mu$-gradient.

For more details on the various possible extensions of the classical theory of the Sobolev spaces to the setting of metric measure spaces, we refer to [Hei07, 10-14] (see also [Che99, Sha00, GT01, Haj03).

The following proposition (whose proof is partly given in AHM15, Proposition 2.28], nevertheless, for completeness we give a proof in \$7) provides useful properties for dealing with calculus of variations in the metric measure setting.

Proposition 1. Under the hypotheses of Theorem 1, we have:

(i) $X$ satisfies the Vitali covering theorem, i.e. for every $A \subset X$ and every family $\mathcal{F}$ of closed balls in $X$, if inf $\left\{\rho>0: \bar{B}_{\rho}(x) \in \mathcal{F}\right\}=0$ for all $x \in A$ (we say that $\mathcal{F}$ is a fine cover of $A$ ) then there exists a countable disjoint subfamily $\mathcal{G}$ of $\mathcal{F}$ such that $\mu\left(A \backslash \bigcup_{B \in \mathcal{G}} B\right)=0$; in other words, $A \subset\left(\bigcup_{B \in \mathcal{G}} B\right) \cup N$ with $\mu(N)=0$;

(ii) the $\mu$-gradient is closable in $H_{\mu}^{1, p}\left(X ; \mathbb{R}^{m}\right)$, i.e. for every $u \in H_{\mu}^{1, p}\left(X ; \mathbb{R}^{m}\right)$ and every measurable set $A \subset X$, if $u(x)=0$ for $\mu$-a.a. $x \in A$ then $\nabla_{\mu} u(x)=0$ for u-a.a. $x \in A$;

(iii) the metric space $X$ enjoys a p-Sobolev inequality, i.e. there exists $C_{S}>0$ such that

$$
\left(\int_{B_{\rho}(x)}|v|^{p} d \mu\right)^{\frac{1}{p}} \leqslant \rho C_{S}\left(\int_{B_{\rho}(x)}\left|\nabla_{\mu} v\right|^{p} d \mu\right)^{\frac{1}{p}}
$$

for all $0<\rho \leqslant \rho_{0}$, with $\rho_{0}>0$, and all $v \in H_{\mu, 0}^{1, p}\left(B_{\rho}(x) ; \mathbb{R}^{m}\right)$, where, for each $O \in \mathcal{O}(X), H_{\mu, 0}^{1, p}\left(O ; \mathbb{R}^{m}\right)$ is the closure of $\operatorname{Lip}_{0}\left(O ; \mathbb{R}^{m}\right)$ with respect to $H_{\mu}^{1, p}$-norm defined in (2.4) with

$$
\operatorname{Lip}_{0}\left(O ; \mathbb{R}^{m}\right):=\left\{u \in \operatorname{Lip}\left(X ; \mathbb{R}^{m}\right): u=0 \text { on } X \backslash O\right\} ;
$$

(iv) for every $u \in H_{\mu}^{1, p}\left(X ; \mathbb{R}^{m}\right)$ and $\mu$-a.e. $x \in X$ there exists $u_{x} \in H_{\mu}^{1, p}\left(X ; \mathbb{R}^{m}\right)$ given by $u_{x}(y):=u(x)+\nabla_{\mu} u(x) \cdot\left(\gamma^{k}(y)-\gamma^{k}(x)\right)$ such that

$$
\begin{array}{r}
\nabla_{\mu} u_{x}(y)=\nabla_{\mu} u(x) \text { for } \mu \text {-a.a. } y \in X ; \\
\lim _{\rho \rightarrow 0} \frac{1}{\rho}\left(f_{B_{\rho}(x)}\left|u(y)-u_{x}(y)\right|^{p} d \mu(y)\right)^{\frac{1}{p}}=0 ;
\end{array}
$$

(v) for every $x \in X$, every $\rho>0$ and every $\tau \in] 0,1[$ there exists a function $\varphi \in$ $\operatorname{Lip}(X ;[0,1])$ such that

$$
\begin{aligned}
& \varphi(x)=0 \text { for all } x \in X \backslash B_{\rho}(x), \varphi(x)=1 \text { for all } x \in \bar{B}_{\tau \rho}(x) \\
& \text { and }\left\|D_{\mu} \varphi\right\|_{L_{\mu}^{\infty}\left(X ; \mathbb{R}^{N}\right)} \leqslant \frac{C_{0}}{\rho(1-\tau)} \text { for some } C_{0}>0 .
\end{aligned}
$$

Such $a \varphi$ is called an Urysohn function for the pair $\left(X \backslash B_{\rho}(x), \bar{B}_{\tau \rho}(x)\right)$.

3. $H_{\mu}^{1, p}$-QUASICONVEXITY IS A NECESSARY CONDITION FOR LOWER SEMICONTINUITY

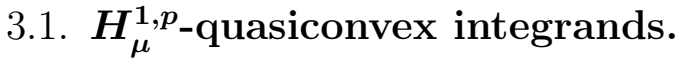

Definition 4. Let $L: X \times \mathbb{M} \longrightarrow]-\infty, \infty]$ be a Borel measurable function. Let $A$ be a measurable subset of $X$. 
(a) We say that $L$ is $H_{\mu}^{1, p}$-quasiconvex on $A$ at $\xi \in \mathbb{M}$ if for $\mu$-a.e. $x \in A$ it holds

$$
L(x, \xi) \leqslant \underline{\lim _{\rho \rightarrow 0}} \inf _{\varphi \in H_{\mu, 0}^{1, p}\left(B_{\rho}(x) ; \mathbb{R}^{m}\right)} f_{\bar{B}_{\rho}(x)} L\left(y, \xi+\nabla_{\mu} \varphi\right) d \mu .
$$

When $L$ is $H_{\mu}^{1, p}$-quasiconvex on $A$ at every $\xi \in \mathbb{M}$ we say that $L$ is $H_{\mu}^{1, p}$-quasiconvex on $A$, and if $A=X$ we say that $L$ is $H_{\mu}^{1, p}$-quasiconvex.

(b) We say that $L$ is Lip-quasiconvex on $A$ at $\xi \in \mathbb{M}$ if for $\mu$-a.e. $x \in A$

$$
L(x, \xi) \leqslant \frac{\lim }{\rho \rightarrow 0} \inf _{\varphi \in \operatorname{Lip}_{0}\left(B_{\rho}(x) ; \mathbb{R}^{m}\right)} f_{\bar{B}_{\rho}(x)} L\left(y, \xi+\nabla_{\mu} \varphi\right) d \mu .
$$

When $L$ is Lip-quasiconvex on $A$ at every $\xi \in \mathbb{M}$ we say that $L$ is Lip-quasiconvex on $A$, and if $A=X$ we say that $L$ is Lip-quasiconvex.

In the following, when we write $L: X \times \mathrm{M} \longrightarrow]-\infty, \infty]$ is a Borel measurable integrand or is an integrand, it simply means that $L$ is a Borel measurable function.

\section{Remarks 1.}

(a) The sum and the max of two $H_{\mu}^{1, p}$-quasiconvex integrands is $H_{\mu}^{1, p}$-quasiconvex.

(b) In the Euclidean setting $(X, d, \mu)=\left(\Omega,|\cdot-\cdot|, \mathcal{L}_{N}\lfloor\Omega)\right.$, by a change of variables, we have

$$
\inf _{\varphi \in W_{0}^{1, p}\left(B_{\rho}(x) ; \mathbb{R}^{m}\right)} f_{\bar{B}_{\rho}(x)} L(\xi+\nabla \varphi) d \mathcal{L}_{N}=\inf _{\varphi \in W_{0}^{1, p}\left(Y ; \mathbb{R}^{m}\right)} \int_{\bar{Y}} L(\xi+\nabla \varphi) d \mathcal{L}_{N},
$$

for all $(x, \xi) \in \Omega \times \mathbb{M}$ and all $\rho>0$, where $L: \mathbb{M} \longrightarrow[0, \infty]$ is Borel measurable and $\bar{Y}=\left\{y \in \mathbb{R}^{N}:|y| \leqslant 1\right\}$. It means that $L$ is $W^{1, p}$-quasiconvex if and only if it is $H_{\mathcal{L}_{N}}^{1, p}$-quasiconvex.

The following lemma is a direct consequence of the Lebesgue differentiation theorem and the definition of $H_{\mu}^{1, p}$-quasiconvexity.

Lemma 1. An integrand $L: X \times \mathbb{M} \longrightarrow[0, \infty]$ is $H_{\mu}^{1, p}$-quasiconvex (resp. Lip-quasiconvex) at $\xi \in \mathbb{M}$ satisfying $L(\cdot, \xi) \in L_{\mu, \text { loc }}^{1}(X)$ if and only if for $\mu$-a.e. $x \in X$

$$
\begin{array}{r}
L(x, \xi)=\lim _{\rho \rightarrow 0} \inf _{\varphi \in H_{\mu, 0}^{1, p}\left(B_{\rho}(x) ; \mathbb{R}^{m}\right)} f_{\bar{B}_{\rho}(x)} L\left(y, \xi+\nabla_{\mu} \varphi\right) d \mu . \\
\left(\operatorname{resp.} L(x, \xi)=\lim _{\rho \rightarrow 0} \inf _{\varphi \in \operatorname{Lip}_{0}\left(B_{\rho}(x) ; \mathbb{R}^{m}\right)} f_{\bar{B}_{\rho}(x)} L\left(y, \xi+\nabla_{\mu} \varphi\right) d \mu .\right)
\end{array}
$$

The following result shows that for Carathéodory integrands with $p$-polynomial growth, Lip-quasiconvexity is equivalent to $H_{\mu}^{1, p}$-quasiconvexity. We follow the proof of [BM84, proposition 2.4 (i), pp. 229] with the necessary changes.

Lemma 2. Let $L: X \times \mathbb{M} \longrightarrow[0, \infty]$ be a Carathéodory integrand, i.e. $L(\cdot, \xi)$ is Borel measurable for all $\xi \in \mathbb{M}$ and $L(x, \cdot)$ is continuous for all $x \in X$. Assume that there exist $C>0$ and $A \in L_{\mu, \text { loc }}^{1}(X)$ such that for $\mu$-a.e. $x \in X$

$$
L(x, \xi) \leqslant A(x)+C\left(1+|\xi|^{p}\right) \quad \text { for all } \xi \in \mathbb{M} .
$$

Then $L$ is Lip-quasiconvex at $\xi \in \mathbb{M}$ if and only if $L$ is $H_{\mu}^{1, p}$-quasiconvex at $\xi$.

Proof. Since $\operatorname{Lip}_{0}\left(B ; \mathbb{R}^{m}\right) \subset H_{\mu, 0}^{1, p}\left(B ; \mathbb{R}^{m}\right)$ for all ball $B, H_{\mu}^{1, p}$-quasiconvexity entails Lipquasiconvexity. 
Now, assume that $L$ is Lip-quasiconvex at $\xi \in \mathbb{M}$. Using the growth condition (3.1), by Lemma 1 we have for $\mu$-a.e. $x \in X$

$$
\lim _{\rho \rightarrow 0} \inf _{\varphi \in \operatorname{Lip}_{0}\left(B_{\rho}(x) ; \mathbb{R}^{m}\right)} f_{\bar{B}_{\rho}(x)} L\left(y, \xi+\nabla_{\mu} \varphi\right) d \mu=L(x, \xi) .
$$

Fix $x \in X$ such that 3.2 holds. Let $\varepsilon>0$. There exists $r_{\varepsilon}>0$ such that for every $\rho \in] 0, r_{\varepsilon}[$ we have

$$
\varepsilon+\inf _{\varphi \in \operatorname{Lip}_{0}\left(B_{\rho}(x) ; \mathbb{R}^{m}\right)} f_{\bar{B}_{\rho}(x)} L\left(y, \xi+\nabla_{\mu} \varphi\right) d \mu \geqslant L(x, \xi) .
$$

Let $\rho \in] 0, r_{\varepsilon}\left[\right.$ and let $\varphi \in H_{\mu, 0}^{1, p}\left(B_{\rho}(x) ; \mathbb{R}^{m}\right)$. By definition of $H_{\mu, 0}^{1, p}\left(B_{\rho}(x) ; \mathbb{R}^{m}\right)$ (see Proposition 1 (ii)), there exists a sequence $\left\{\varphi_{n}\right\}_{n \in \mathbb{N}} \subset \operatorname{Lip}_{0}\left(B_{\rho}(x) ; \mathbb{R}^{m}\right)$ such that $\nabla_{\mu} \varphi_{n}(\cdot) \longrightarrow$ $\nabla_{\mu} \varphi(\cdot) \mu$-a.e. in $X$ and $\lim _{n \rightarrow \infty}\left\|\nabla_{\mu} \varphi_{n}-\nabla_{\mu} \varphi\right\|_{L_{\mu}^{p}\left(B_{\rho}(x) ; \mathbb{M}\right)}=0$. By Fatou lemma we have

$$
\begin{aligned}
\varliminf_{n \rightarrow \infty} f_{\bar{B}_{\rho}(x)} A(y)+C(1 & \left.+\left|\xi+\nabla_{\mu} \varphi_{n}\right|^{p}\right)-L\left(y, \xi+\nabla_{\mu} \varphi_{n}\right) d \mu \\
& \geqslant f_{\bar{B}_{\rho}(x)} A(y)+C\left(1+\left|\xi+\nabla_{\mu} \varphi\right|^{p}\right)-L\left(y, \xi+\nabla_{\mu} \varphi\right) d \mu
\end{aligned}
$$

On the other side, using $(3.3)$ we have

$$
\begin{aligned}
& \varliminf_{n \rightarrow \infty} f_{\bar{B}_{\rho}(x)} A(y)+C\left(1+\left|\xi+\nabla_{\mu} \varphi_{n}\right|^{p}\right)-L\left(y, \xi+\nabla_{\mu} \varphi_{n}\right) d \mu \\
& \leqslant f_{\bar{B}_{\rho}(x)} A(y)+C\left(1+\left|\xi+\nabla_{\mu} \varphi\right|^{p}\right) d \mu-\inf _{\varphi \in \operatorname{Lip}_{0}\left(B_{\rho}(x) ; \mathbb{R}^{m}\right)} f_{\bar{B}_{\rho}(x)} L\left(y, \xi+\nabla_{\mu} \varphi\right) d \mu \\
& \leqslant f_{\bar{B}_{\rho}(x)} A(y)+C\left(1+\left|\xi+\nabla_{\mu} \varphi\right|^{p}\right) d \mu-L(x, \xi)+\varepsilon .
\end{aligned}
$$

Collecting (3.4) and (3.5) we obtain

$$
-\varepsilon+L(x, \xi) \leqslant f_{\bar{B}_{\rho}(x)} L\left(y, \xi+\nabla_{\mu} \varphi\right) d \mu .
$$

Taking the infimum over all $\varphi \in H_{\mu, 0}^{1, p}\left(B_{\rho}(x) ; \mathbb{R}^{m}\right)$ and then over all $\left.\rho \in\right] 0, r_{\varepsilon}[$ gives

$$
-\varepsilon+L(x, \xi) \leqslant \varliminf_{\rho \rightarrow 0} \operatorname{limf}_{\varphi \in H_{\mu, 0}^{1, p}\left(B_{\rho}(x) ; \mathbb{R}^{m}\right)} f_{\bar{B}_{\rho}(x)} L\left(y, \xi+\nabla_{\mu} \varphi\right) d \mu,
$$

and the proof is finished by letting $\varepsilon \rightarrow 0$.

3.2. Necessary condition for the lower semicontinuity. Here we show that $H_{u}^{1, p_{-}}$ quasiconvexity is a necessary condition for the lower semicontinuity of the integrals (1.1) with respect to the strong convergence of $L_{\mu}^{p}\left(X ; \mathbb{R}^{m}\right)$. The proof is inspired by the proof of Cheeger [Che99, Theorem 3.7] that a Lipschitz function, defined on a metric measure space with doubling measure and satisfying a $(1, p)$-Poincaré inequality, is asymptotically generalized linear; this was a fundamental step towards setting up a differential structure on metric measure spaces.

Theorem 2. Let $L: X \times \mathbb{M} \longrightarrow[0, \infty]$ be a p-coercive Borel measurable integrand, i.e. satisfying for some $c>0$, for $\mu$-a.e. $x \in X$ and for every $\xi \in \mathbb{M}$

$$
L(x, \xi) \geqslant c|\xi|^{p}
$$


Assume that for every $u,\left\{u_{\varepsilon}\right\}_{\varepsilon>0} \subset H_{\mu}^{1, p}\left(X ; \mathbb{R}^{m}\right)$ satisfying $\lim _{\varepsilon \rightarrow 0}\left\|u_{\varepsilon}-u\right\|_{L_{\mu}^{p}\left(X ; \mathbb{R}^{m}\right)}=0$, it holds

$$
\varliminf_{\varepsilon \rightarrow 0} \int_{X} L\left(x, \nabla_{\mu} u_{\varepsilon}\right) d \mu \geqslant \int_{X} L\left(x, \nabla_{\mu} u\right) d \mu .
$$

Then $L$ is $H_{\mu}^{1, p}$-quasiconvex at every $\xi \in \mathbb{M}$ satisfying $L(\cdot, \xi) \in L_{\mu}^{1}(X)$.

Proof. Let $\xi \in \mathbb{M}$ be such that $L(\cdot, \xi) \in L_{\mu}^{1}(X)$. By Lebesgue differentiation theorem we have for $\mu$-a.e. $x \in X$

$$
\lim _{\rho \rightarrow 0} f_{\bar{B}_{\rho}(x)} L(y, \xi) d \mu=L(x, \xi)<\infty .
$$

Let $\alpha>0$. We have to show that $\mu\left(N_{\alpha}\right)=0$ where

$$
N_{\alpha}:=\left\{x \in X: \lim _{\rho \rightarrow 0} \inf _{\varphi \in H_{\mu, 0}^{1, p}\left(B_{\rho}(x) ; \mathbb{R}^{m}\right)} f_{\bar{B}_{\rho}(x)} L\left(y, \xi+\nabla_{\mu} \varphi\right)-L(y, \xi) d \mu<-\alpha\right\} .
$$

Since $X$ is a countable union of balls with finite measure (see HKST15, Lemma 3.3.28, pp. 62]), we can write

$$
N_{\alpha}=\bigcup_{s=0}^{\infty} B_{s} \cap N_{\alpha} \quad \text { where } B_{s} \text { is an open ball and } \mu\left(B_{s}\right)<\infty,
$$

so it is enough to prove that $\mu\left(N_{\alpha}^{s}\right)=0$ where $N_{\alpha}^{s}:=B_{s} \cap N_{\alpha}$ for all $s \in \mathbb{N}$.

Fix $s \in \mathbb{N}$. For each $x \in N_{\alpha}^{s}$ there exists $\left.\rho^{x} \in\right] 0,1\left[\operatorname{such}\right.$ that $\bar{B}_{\rho^{x}}(x) \subset B_{s}$. Fix $(k, n) \in \mathbb{N}^{*} \times \mathbb{N}^{*}$. For each $x \in N_{\alpha}^{s}$ there exist $\left.\rho_{k, n}^{x} \in\right] 0, \min \left(\left(\frac{1}{k 2^{n}}\right)^{\frac{1}{p}}, \rho^{x}\right)\left[\right.$ and $\varphi_{k, n}^{x} \in$ $H_{\mu, 0}^{1, p}\left(B_{\rho_{k, n}^{x}}(x) ; \mathbb{R}^{m}\right)$ such that

$$
f_{\bar{B}_{\rho_{k, n}^{x}}(x)} L\left(y, \xi+\nabla_{\mu} \varphi_{k, n}^{x}\right)-L(y, \xi) d \mu<-\alpha+\frac{1}{k \mu\left(B_{s}\right)} .
$$

The family of closed balls $\left\{\bar{B}_{\rho_{k, n}^{x}}(x)\right\}_{x \in N_{\alpha}^{s}, n \in \mathbb{N}^{*}}$ is a fine cover of $N_{\alpha}^{s}$, so by Vitali covering theorem there exists a countable family of mutually disjoint closed balls $\left\{\bar{B}_{\rho_{k, n_{i}}^{x_{i}}}\left(x_{i}\right)\right\}_{i \in \mathbb{N}}$ such that

$$
\mu\left(N_{\alpha}^{s} \backslash \bigcup_{i=0}^{\infty} \bar{B}_{\rho_{k, n_{i}}^{x_{i}}}\left(x_{i}\right)\right)=0 \quad \text { and } \bigcup_{i=0}^{\infty} \bar{B}_{\rho_{k, n_{i}}^{x_{i}}}\left(x_{i}\right) \subset B_{s} .
$$

We set $\varphi_{k}^{i}:=\varphi_{k, n_{i}}^{x_{i}}, B_{k}^{i}:=\bar{B}_{\rho_{k, n_{i}}^{x_{i}}}\left(x_{i}\right), \rho_{k}^{i}:=\rho_{k, n_{i}}^{x_{i}}$ for all $i \in \mathbb{N}$. Moreover, for each $l \in \mathbb{N}$ we set $\varphi_{k, l}:=\sum_{i=0}^{l} \varphi_{k}^{i} \mathbb{1}_{B_{k}^{i}} \in H_{\mu, 0}^{1, p}\left(B_{s} ; \mathbb{R}^{m}\right)$. Assume for the moment that

$$
\lim _{k \rightarrow \infty}\left\|\varphi_{k, l}\right\|_{L_{\mu}^{p}\left(X ; \mathbb{R}^{m}\right)}=0 \quad \text { for all } l \in \mathbb{N} .
$$

Fix $l \in \mathbb{N}$. Set $F_{k}^{l}:=\bigcup_{i=0}^{l} B_{k}^{i} \subset B_{s}$. Let $\varepsilon>0$. Using (3.9) we can assert that there exists $l_{\varepsilon} \in \mathbb{N}$ such that $\mu\left(N_{\alpha}^{s} \backslash F_{k}^{l_{\varepsilon}}\right)<\frac{\varepsilon}{\alpha}$. Now, we take the sum over the finite family of closed balls $\left\{B_{k}^{i}\right\}_{i=0}^{l_{\varepsilon}}$ in 3.8

$$
\int_{F_{k}^{l_{\varepsilon}}} L\left(y, \xi+\nabla_{\mu} \varphi_{k, l_{\varepsilon}}\right)-L(y, \xi) d \mu \leqslant \frac{1}{k}-\alpha \mu\left(F_{k}^{l_{\varepsilon}}\right) .
$$


For each $i \in \mathbb{N}$ we have $\varphi_{k}^{i}=0 \mu$-a.e. in $X \backslash B_{\rho_{k, n_{i}}^{x_{i}}}\left(x_{i}\right)$ and thus $\varphi_{k}^{i}=0 \mu$-a.e. in $X \backslash B_{k}^{i}$ where $B_{k}^{i}=\bar{B}_{\rho_{k, n_{i}}^{x_{i}}}\left(x_{i}\right)$. It follows from Proposition 1 (ii) that $L\left(\cdot, \xi+\nabla_{\mu} \varphi_{k, l_{\varepsilon}}\right)-L(\cdot, \xi)=$ $0 \mu$-a.e. in $X \backslash F_{k}^{l_{\varepsilon}}$, and taking account of $L(\cdot, \xi) \in L_{\mu}^{1}(X)$ we have

$$
\int_{X} L\left(y, \xi+\nabla_{\mu} \varphi_{k, l_{\varepsilon}}\right)-L(y, \xi) d \mu=\int_{F_{k}^{l_{\varepsilon}}} L\left(y, \xi+\nabla_{\mu} \varphi_{k, l_{\varepsilon}}\right)-L(y, \xi) d \mu \leqslant \frac{1}{k}-\alpha \mu\left(N_{\alpha}^{s}\right)+\varepsilon .
$$

Using lower semicontinuity of the integral (3.7) and letting $k \rightarrow \infty$ in 3.10 we have

$$
0 \leqslant \varliminf_{k \rightarrow \infty} \int_{X} L\left(y, \xi+\nabla_{\mu} \varphi_{k, l_{\varepsilon}}\right)-L(y, \xi) d \mu \leqslant-\alpha \mu\left(N_{\alpha}^{s}\right)+\varepsilon .
$$

Letting $\varepsilon \rightarrow 0$, we deduce $\mu\left(N_{\alpha}^{s}\right)=0$ which completes the proof.

It remains to show that the sequence $\left\{\varphi_{k, l}\right\}_{k}$ is converging to 0 in $L_{\mu}^{p}\left(X ; \mathbb{R}^{m}\right)$. Using the Sobolev inequality (2.5), the coercivity condition 3.6 and inequality 3.8 we have

$$
\begin{aligned}
\int_{X}\left|\varphi_{k, l}(y)\right|^{p} d \mu & =\sum_{i=0}^{l} \int_{B_{k}^{i}}\left|\varphi_{k}^{i}(y)\right|^{p} d \mu \\
& \leqslant \sum_{i=0}^{\infty}\left(\rho_{k}^{i}\right)^{p} C_{S}^{p} \int_{B_{k}^{i}}\left|\nabla_{\mu} \varphi_{k}^{i}(y)\right|^{p} d \mu \\
& \leqslant \frac{2^{p-1} C_{S}^{p}}{c} \sum_{i=0}^{\infty}\left(\rho_{k}^{i}\right)^{p} \int_{B_{k}^{i}} L\left(y, \xi+\nabla_{\mu} \varphi_{k}^{i}(y)\right)+L(y, \xi) d \mu \\
& \leqslant \frac{2^{p-1} C_{S}^{p}}{c} \sum_{i=0}^{\infty}\left(\rho_{k}^{i}\right)^{p} \int_{B_{k}^{i}}-\alpha+\frac{1}{k \mu\left(B_{s}\right)}+2 L(y, \xi) d \mu \\
& \leqslant\left(\frac{2^{p-1} C_{S}^{p}}{c} \int_{B_{s}}-\alpha+\frac{1}{k \mu\left(B_{s}\right)}+2 L(y, \xi) d \mu\right) \frac{2}{k} .
\end{aligned}
$$

Since $L(\cdot, \xi) \in L_{\mu}^{1}\left(B_{s}\right)$, by passing to the limit $k \rightarrow \infty$ we obtain $\varphi_{k, l} \longrightarrow 0$ in $L_{\mu}^{p}\left(X ; \mathbb{R}^{m}\right)$.

Remark 1. In the proof of Theorem 2 the finitness of $\int_{X} L(y, \xi) d \mu$ is used only in the last step of the proof of Theorem 2 , before that, it is sufficient to assume that

$$
\int_{B} L(y, \xi) d \mu<\infty \text { for all open ball } B \subset X \text { with } \mu(B)<\infty \text {. }
$$

In fact, if we replace 3.10$)$ with

$\int_{B_{s}} L\left(y, \xi+\nabla_{\mu} \varphi_{k, l_{\varepsilon}}\right)-L(y, \xi) d \mu=\int_{F_{k}^{l_{\varepsilon}}} L\left(y, \xi+\nabla_{\mu} \varphi_{k, l_{\varepsilon}}\right)-L(y, \xi) d \mu \leqslant \frac{1}{k}-\alpha \mu\left(N_{\alpha}^{s}\right)+\varepsilon$

and if we assume that the lower semicontinuity of the integrals holds for every open set with finite measure, then we can conclude that $L$ is $H_{\mu}^{1, p}$-quasiconvex at every $\xi \in \mathbb{M}$ satisfying $\int_{B} L(y, \xi) d \mu<\infty$ for every open ball $B$ with finite measure.

Denote by $L_{\mu, \text { loc* }}^{1}(X)$ the space of all measurable functions $u: X \longrightarrow \overline{\mathbb{R}}$ such that

$$
\int_{B}|u| d \mu<\infty \quad \text { for all open ball } B \subset X \text { satisfying } \mu(B)<\infty \text {. }
$$

Remark 1 leads to a "local version" of Theorem 2 , 
Theorem 3. Let $L: X \times \mathbb{M} \longrightarrow[0, \infty]$ be a p-coercive Borel measurable integrand. Assume that for every $u,\left\{u_{\varepsilon}\right\}_{\varepsilon>0} \subset H_{\mu}^{1, p}\left(X ; \mathbb{R}^{m}\right)$ satisfying $\lim _{\varepsilon \rightarrow 0}\left\|u_{\varepsilon}-u\right\|_{L_{\mu}^{p}\left(X ; \mathbb{R}^{m}\right)}=0$, it holds

$$
\varliminf_{\varepsilon \rightarrow 0} \int_{O} L\left(x, \nabla_{\mu} u_{\varepsilon}\right) d \mu \geqslant \int_{O} L\left(x, \nabla_{\mu} u\right) d \mu
$$

for all open set $O \in \mathcal{O}(X)$ with $\mu(O)<\infty$.

Then $L$ is $H_{\mu}^{1, p}$-quasiconvex on $X$ at every $\xi \in \mathbb{M}$ satisfying $L(\cdot, \xi) \in L_{\mu, \text { loc* }}^{1}(X)$.

For a Borel measurable integrand $L: X \times \mathrm{M} \longrightarrow]-\infty, \infty]$, we say that $L$ is convex and lower semicontinuous if for $\mu$-a.e. $x \in X$ the integrand $L(x, \cdot): \mathbb{M} \longrightarrow]-\infty, \infty]$ is convex and lower semicontinuous. We show in Proposition 2 below that a convex and lower semicontinuous integrand is $H_{\mu}^{1, p}$-quasiconvex. The proof is indirect, we deduce the $H_{\mu}^{1, p}$-quasiconvexity from the lower semicontinuity of the convex integral functional associated with $L$. If $L$ does not depend on $x$, it is direct to see that a convex and lower semicontinuous integrand $L$ is $H_{\mu}^{1, p}$-quasiconvex; see $\$ 5$ for related discussion.

Proposition 2. Let $L: X \times \mathbb{M} \longrightarrow$ ] $-\infty, \infty]$ be a $p$-coercive, convex and lower semicontinuous (Borel measurable) integrand. Then $L$ is $H_{\mu}^{1, p}$-quasiconvex on $X$ at every $\xi \in \mathbb{M}$ satisfying $L(\cdot, \xi) \in L_{\mu, \mathrm{loc}}^{1}(X)$.

Proof. Define the integral functional $I: H_{\mu}^{1, p}\left(X ; \mathbb{R}^{m}\right) \times \mathcal{O}(X) \longrightarrow[0, \infty]$ by

$$
I(u ; O):=\int_{O} L\left(x, \nabla_{\mu} u\right) d \mu .
$$

Let $u,\left\{u_{n}\right\}_{n \in \mathbb{N}} \subset H_{\mu}^{1, p}\left(X ; \mathbb{R}^{m}\right)$ satisfying $u_{n} \longrightarrow u$ in $L_{\mu}^{p}\left(X ; \mathbb{R}^{m}\right)$ as $n \rightarrow \infty$. Let $O \in \mathcal{O}(X)$ be an open set. If $\underline{\lim }_{n \rightarrow \infty} I\left(u_{n} ; O\right)<\infty$ then for a subsequence (not relabelled) of $\left\{u_{n}\right\}_{n \in \mathbb{N}}$ we have $\sup _{n \in \mathbb{N}} I\left(u_{n} ; O\right)<\infty$ and $\underline{\lim }_{n \rightarrow \infty} I\left(u_{n} ; O\right)=\lim _{n \rightarrow \infty} I\left(u_{n} ; O\right)$. Hence $\sup _{n \in \mathbb{N}}\left\|\nabla_{\mu} u_{n}\right\|_{L_{\mu}^{p}(O ; \mathbb{M})}<\infty$ since $L$ is $p$-coercive. By the reflexivity of $H_{\mu}^{1, p}\left(O ; \mathbb{R}^{m}\right)$, there exists a subsequence $\left\{u_{n}\right\}_{n \in \mathbb{N}}$ such that $\nabla_{\mu} u_{n} \rightarrow \nabla_{\mu} u$ in $L_{\mu}^{p}(O ; \mathbb{M})$ as $n \rightarrow \infty$. The integral $I(\cdot ; O)$ is convex since $L$ is a convex integrand. Moreover, $I(\cdot ; O)$ is lower semicontinuous with respect to the strong convergence of $H_{\mu}^{1, p}\left(O ; \mathbb{R}^{m}\right)$ by using Fatou lemma and lower semicontinuity of $L$. So, $I(\cdot ; O)$ is sequentially weakly lower semicontinuous on $H_{\mu}^{1, p}\left(O ; \mathbb{R}^{m}\right)$, that is $\underline{\lim }_{n \rightarrow \infty} I\left(u_{n} ; O\right) \geqslant I(u ; O)$. The proof is finished by applying Theorem 3 ,

3.3. Abstract version of Theorem 3. Let us denote by $\mathcal{B}(X)$ the family of Borel sets of $X$. For a functional $I: H_{\mu}^{1, p}\left(X ; \mathbb{R}^{m}\right) \times \mathcal{B}(X) \longrightarrow[0, \infty]$ we consider the following conditions:

$\left(\mathrm{C}_{1}\right)$ for each $u \in H_{\mu}^{1, p}\left(X ; \mathbb{R}^{m}\right)$ the set function $I(u ; \cdot)$ is a Borel measure absolutely continuous with respect to $\mu$;

$\left(\mathrm{C}_{2}\right)$ there exists $c>0$ such that $I(v ; B) \geqslant c\left\|\nabla_{\mu} v\right\|_{L_{\mu}^{p}(B ; \mathbb{M})}^{p}$ for all $v \in H_{\mu}^{1, p}\left(X ; \mathbb{R}^{m}\right)$ and all closed balls $B$ with $\mu(B)<\infty ;(I(\cdot ; B)$ is a $p$-coercive functional $)$

$\left(\mathrm{C}_{3}\right)$ for every closed ball $B$ and every $(u, v) \in H_{\mu}^{1, p}\left(X ; \mathbb{R}^{m}\right)^{2}$ if $u=v \mu$-a.e. in $B$ then $I(u ; B)=I(v ; B) ;(I$ is a local functional on closed balls $)$

$\left(\mathrm{C}_{4}\right)$ for every open set $O \in \mathcal{O}(X)$ with $\mu(O)<\infty$ and every $(u, v) \in H_{\mu}^{1, p}\left(X ; \mathbb{R}^{m}\right)^{2}$ if $u=v \mu$-a.e. in $O$ then $I(u ; O)=I(v ; O)$. ( $I$ is a local functional on open sets)

For each $u \in H_{\mu}^{1, p}\left(X ; \mathbb{R}^{m}\right)$ we consider the assertion:

$\left(\mathrm{C}_{u}\right)$ for every $O \in \mathcal{O}(X)$ with $\mu(O)<\infty$ the functional $I(\cdot ; O)$ is $L_{\mu}^{p}$-lower semicontinuous at $u$, i.e. for every $\left\{u_{\varepsilon}\right\}_{\varepsilon>0} \subset H_{\mu}^{1, p}\left(X ; \mathbb{R}^{m}\right)$ with $\lim _{\varepsilon \rightarrow 0}\left\|u_{\varepsilon}-u\right\|_{L_{\mu}^{p}\left(X ; \mathbb{R}^{m}\right)}=0$ it 
holds

$$
\varliminf_{\varepsilon \rightarrow 0} I\left(u_{\varepsilon} ; O\right) \geqslant I(u ; O) .
$$

Using arguments similar to those of the proof of Theorem 2 we can write an abstract version of Theorem 3; for completeness the proof is given in $\$ 7$.

Theorem 4. Assume that $\left(\mathrm{C}_{1}\right),\left(\mathrm{C}_{2}\right),\left(\mathrm{C}_{3}\right)$, and $\left(\mathrm{C}_{4}\right)$ hold. Let $u \in H_{\mu}^{1, p}\left(X ; \mathbb{R}^{m}\right)$ be such that $\left(\mathrm{C}_{u}\right)$ holds. If

$$
I(u ; B)<\infty \text { for all open ball } B \subset X \text { with } \mu(B)<\infty,
$$

then for $\mu$-a.e. $x \in X$

$$
\frac{d I(u ; \cdot)}{d \mu}(x)=\lim _{\rho \rightarrow 0} \frac{I\left(u ; \bar{B}_{\rho}(x)\right)}{\mu\left(\bar{B}_{\rho}(x)\right)}=\lim _{\rho \rightarrow 0} \inf _{\varphi \in H_{\mu, 0}^{1, p}\left(B_{\rho}(x) ; \mathbb{R}^{m}\right)} \frac{I\left(u+\varphi ; \bar{B}_{\rho}(x)\right)}{\mu\left(\bar{B}_{\rho}(x)\right)} .
$$

The following consequence justifies the introduction of the condition $\left(\mathcal{Q}_{L}\right)$ below (see $\$ 4.1$, which will play the role of $H_{\mu}^{1, p}$-quasiconvexity for integrands depending on the triple $(x, v, \xi)$.

Corollary 1. Let $L: X \times \mathbb{R}^{m} \times \mathrm{M} \longrightarrow[0, \infty]$ be a p-coercive Borel measurable integrand. Assume that for every $u,\left\{u_{\varepsilon}\right\}_{\varepsilon>0} \subset H_{\mu}^{1, p}\left(X ; \mathbb{R}^{m}\right)$ satisfying $\lim _{\varepsilon \rightarrow 0}\left\|u_{\varepsilon}-u\right\|_{L_{\mu}^{p}\left(X ; \mathbb{R}^{m}\right)}=0$, it holds

$$
\varliminf_{\varepsilon \rightarrow 0} \int_{O} L\left(x, u_{\varepsilon}, \nabla_{\mu} u_{\varepsilon}\right) d \mu \geqslant \int_{O} L\left(x, u, \nabla_{\mu} u\right) d \mu
$$

for all open set $O \in \mathcal{O}(X)$ satisfying $\mu(O)<\infty$.

Let $\bar{u} \in H_{\mu}^{1, p}\left(X ; \mathbb{R}^{m}\right)$ be such that $L\left(\cdot, \bar{u}(\cdot), \nabla_{\mu} \bar{u}(\cdot)\right) \in L_{\mu, \mathrm{loc}^{*}}^{1}(X)$. Then for $\mu$-a.e. $x \in X$

$$
L\left(x, \bar{u}(x), \nabla_{\mu} \bar{u}(x)\right)=\lim _{\rho \rightarrow 0} \inf _{\varphi \in H_{\mu, 0}^{1, p}\left(B_{\rho}(x) ; \mathbb{R}^{m}\right)} f_{\bar{B}_{\rho}(x)} L\left(y, \bar{u}+\varphi, \nabla_{\mu} \bar{u}+\nabla_{\mu} \varphi\right) d \mu .
$$

Proof. Define $I: H_{\mu}^{1, p}\left(X ; \mathbb{R}^{m}\right) \times \mathcal{B}(X) \longrightarrow[0, \infty]$ by

$$
I(w ; A):=\int_{A} L\left(y, w, \nabla_{\mu} w\right) d \mu .
$$

The conditions $\left(\mathrm{C}_{3}\right)$ and $\left(\mathrm{C}_{4}\right)$ are satisfied since (ii) of Theorem 1 . It is clear that $\left(\mathrm{C}_{1}\right)$ holds. Since $L\left(\cdot, \bar{u}(\cdot), \nabla_{\mu} \bar{u}(\cdot)\right) \in L_{\mu, \text { loc* }}^{1}(X)$ we have $I(\bar{u} ; B)<\infty$ for all open ball $B \subset X$ with $\mu(B)<\infty$, so $(3.12)$ holds with $u=\bar{u}$. We see that $\left(\mathrm{C}_{2}\right)$ holds since $L$ is $p$-coercive. The condition $\left(\mathrm{C}_{u}\right)$ is satisfied with $u=\bar{u}$ since (3.13). Therefore applying Theorem 4 we obtain (3.14).

\section{4. $H_{\mu}^{1, p}$-QUASICONVEXITY IS A NECESSARY AND SUFFICIENT CONDITION FOR LOWER SEMICONTINUITY UNDER POLYNOMIAL GROWTH}

We say that the metric measure space $(X, d, \mu)$ satisfies the annular decay property (which was introduced independently by [Buc99, pp. 521 and $\S 2$ pp. 524] and [CM98]) if $\left(\mathrm{AD}_{X}\right)$ there exist $\eta>0$ and $K \geqslant 1$ such that for every $x \in X$, every $\rho>0$ and every $\tau \in] 0,1[$,

$$
\mu\left(B_{\rho}(x) \backslash B_{\tau \rho}(x)\right) \leqslant K(1-\tau)^{\eta} \mu\left(B_{\rho}(x)\right) .
$$

The annular decay property holds, for instance, when the metric space is a length space, i.e. metric space in which the distance between points is the infimum of lenghts of rectifiable paths joining those points, see [Buc99, Corollary 2.2], [CM98, Lemma 3.3], [Che99, Proposition 6.12] and [HKST15, Proposition 11.5.3, pp. 328]). We can remark 
that, when the annular decay property holds, the boundary of balls is of zero measure, indeed, if $x \in X, \rho>0$ and $\tau \in] \frac{1}{2}, 1[$, we have

$$
\mu\left(\partial B_{\rho}(x)\right) \leqslant \mu\left(B_{\frac{\rho}{\tau}}(x) \backslash B_{\rho}(x)\right) \leqslant K\left(1-\frac{1}{\tau}\right)^{\eta} \mu\left(B_{\frac{\rho}{\tau}}(x)\right) \leqslant K\left(1-\frac{1}{\tau}\right)^{\eta} \mu\left(B_{2 \rho}(x)\right),
$$

letting $\tau \rightarrow 1$ we obtain that $\mu\left(\partial B_{\rho}(x)\right)=0$.

To prove the sufficiency of $H_{\mu}^{1, p}$-quasiconvexity for the lower semicontinuity, we need the following property of Alexandrov type (or of Portmanteau type)

$\left(\mathrm{AL}_{X}\right)$ for every open set $O \in \mathcal{O}(X)$ and for every sequence $\left\{m_{n}\right\}_{n \in \mathbb{N}}$ of nonnegative Borel regular measures on $O$ satisfying $\sup _{n \in \mathbb{N}} m_{n}(O)<\infty$ there exist a subsequence (not relabelled) $\left\{m_{n}\right\}_{n \in \mathbb{N}}$ and $m$ a locally finite Borel regular measure on $O$ such that

$$
\begin{array}{ll}
\varliminf_{n \rightarrow \infty} m_{n}(V) \geqslant m(V) & \text { for all open set } V \subset O . \\
\varlimsup_{n \rightarrow \infty} m_{n}(B) \leqslant m(B) & \text { for all closed ball } B \subset O .
\end{array}
$$

For instance, if $X$ is compact or locally compact then the property $\left(\mathrm{AL}_{X}\right)$ holds.

\subsection{Sufficiency of $\boldsymbol{H}_{\mu}^{1, p}$-quasiconvexity under polynomial growth conditions.}

In this subsection we prove the sequential weak lower semicontinuity on $H_{\mu}^{1, p}\left(X ; \mathbb{R}^{m}\right)$ of integral functionals under polynomial growth conditions and condition $\left(\mathcal{Q}_{L}\right)$ below.

If $\xi \in \mathbb{M}, v \in \mathbb{R}^{m}$ and $x \in X_{k}$ are given with $k \in \mathbb{N}$, we can find $\Lambda_{x, v, \xi}^{k} \in \operatorname{Lip}\left(X ; \mathbb{R}^{m}\right)$ such that $\nabla_{\mu} \Lambda_{x, v, \xi}^{k}=\xi \mu$-a.e. in $X$ and $\Lambda_{x, v, \xi}^{k}(x)=v$. Indeed, we set $\Lambda_{x, v, \xi}^{k}=\left(\Lambda_{x, v, \xi, i}^{k}\right)_{i=1, \cdots, m}$ with

$$
\Lambda_{x, v, \xi, i}^{k}(y):=v_{i}+\xi_{i \bullet} \cdot\left(\gamma^{k}(y)-\gamma^{k}(x)\right) \text { for } y \in X_{k},
$$

where $\xi_{i \bullet} \in \mathbb{R}^{N}$ is the $i$ th row of $\xi$ and where $\left(X_{k}, \gamma^{k}\right)$ are given by Theorem 1 . Note that $u_{x}=\Lambda_{x, u(x), \nabla_{\mu} u(x)}^{k}$ where $u_{x}$ is given by Proposition 1 (iv).

The following condition will play the role of $H_{\mu}^{1, p}$-quasiconvexity for integrands depending on the tripe $(x, v, \xi)$ :

$\left(\mathcal{Q}_{L}\right)$ for every $(v, \xi) \in \mathbb{R}^{m} \times \mathbb{M}$, for every $k \in \mathbb{N}$ and for $\mu$-a.e. $x \in X_{k}$

$$
L(x, v, \xi) \leqslant \underline{\lim _{\rho \rightarrow 0}} \inf _{\varphi \in H_{\mu, 0}^{1, p}\left(B_{\rho}(x) ; \mathbb{R}^{m}\right)} f_{\bar{B}_{\rho}(x)} L\left(y, \Lambda_{x, v, \xi}^{k}+\varphi, \xi+\nabla_{\mu} \varphi\right) d \mu .
$$

Remark 2. If $L$ does not depend on $v$ then $\left(\mathcal{Q}_{L}\right)$ is nothing but the $H_{\mu}^{1, p}$-quasiconvexity of $L$ at every $\xi \in \mathbb{M}$. We also see that $\left(\mathcal{Q}_{L}\right)$ implies that for $\mu$-a.e. $x \in X_{k}$ and for every $(v, \xi) \in \mathbb{Q}^{m} \times \mathbb{Q}^{m N}$

$$
L(x, v, \xi) \leqslant \underline{\lim _{\rho \rightarrow 0}} \inf _{\varphi \in H_{\mu, 0}^{1, p}\left(B_{\rho}(x) ; \mathbb{R}^{m}\right)} f_{\bar{B}_{\rho}(x)} L\left(y, \Lambda_{x, v, \xi}^{k}+\varphi, \xi+\nabla_{\mu} \varphi\right) d \mu
$$

where $\mathbb{Q}^{m} \subset \mathbb{R}^{m}$ (resp. $\mathbb{Q}^{m N} \subset \mathbb{M}$ ) is the $m$ rows vectors set (resp. the $m$ rows $N$ columns matrices set) with rational number entries.

For a Borel measurable integrand $L: X \times \mathbb{R}^{m} \times \mathbb{M} \longrightarrow[0, \infty]$, we denote by $I$ : $H_{\mu}^{1, p}\left(X ; \mathbb{R}^{m}\right) \times \mathcal{O}(X) \longrightarrow[0, \infty]$ the associated integral functional which is given by

$$
I(u ; O):=\int_{O} L\left(x, u, \nabla_{\mu} u\right) d \mu .
$$

Theorem 5. Assume that $(X, d, \mu)$ satisfies $\left(\mathrm{AD}_{X}\right)$ and $\left(\mathrm{AL}_{X}\right)$, Let $L: X \times \mathbb{R}^{m} \times \mathbb{M} \longrightarrow$ $[0, \infty]$ be a Borel measurable integrand. Assume that 
(i) L satisfies $\left(\mathcal{Q}_{L}\right)$;

(ii) L has p-polynomial growth, i.e. there exist $c, C>0$ and $A \in L_{\mu \text {,loc }}^{1}(X)$ such that for $\mu$-a.e. $x \in X$

$$
L(x, v, \xi) \leqslant A(x)+C\left(1+|v|^{p}+|\xi|^{p}\right) \quad \text { for all }(v, \xi) \in \mathbb{R}^{m} \times \mathbb{M}
$$

(iii) for $\mu$-a.e. $x \in X$ the function $\mathbb{R}^{m} \times \mathbb{M} \ni(v, \xi) \longmapsto L(x, v, \xi)$ is lower semicontinuous.

Then for all open sets $O \in \mathcal{O}(X)$ the integral functional $I(\cdot ; O)$ is sequentially weakly lower semicontinuous on $H_{\mu}^{1, p}\left(X ; \mathbb{R}^{m}\right)$, i.e. for every $u \in H_{\mu}^{1, p}\left(X ; \mathbb{R}^{m}\right)$ and every sequence $\left\{u_{\varepsilon}\right\}_{\varepsilon>0} \subset H_{\mu}^{1, p}\left(X ; \mathbb{R}^{m}\right)$ satisfying $\lim _{\varepsilon \rightarrow 0}\left\|u_{\varepsilon}-u\right\|_{L_{\mu}^{p}\left(X ; \mathbb{R}^{m}\right)}=0$ and $\sup _{\varepsilon>0}\left\|\nabla_{\mu} u_{\varepsilon}\right\|_{L_{\mu}^{p}(X ; \mathrm{M})}^{p}<$ $\infty$, we have

$$
\varliminf_{\varepsilon \rightarrow 0} I\left(u_{\varepsilon} ; O\right) \geqslant I(u ; O) \text { for all open set } O \in \mathcal{O}(X) .
$$

Proof. Let $O \in \mathcal{O}(X)$ be an open set. Let $u,\left\{u_{\varepsilon}\right\}_{\varepsilon>0} \subset H_{\mu}^{1, p}\left(X ; \mathbb{R}^{m}\right)$ be such that $\lim _{\varepsilon \rightarrow 0}\left\|u_{\varepsilon}-u\right\|_{L_{\mu}^{p}\left(X ; \mathbb{R}^{m}\right)}=0$ in $L_{\mu}^{p}\left(X ; \mathbb{R}^{m}\right)$ and $\sup _{\varepsilon>0}\left\|\nabla_{\mu} u_{\varepsilon}\right\|_{L_{\mu}^{p}(X ; \mathbb{M})}^{p}<\infty$. Consider a sequence $\left.\left\{\varepsilon_{n}\right\}_{n \in \mathbb{N}} \subset\right] 0,1\left[\right.$ satisfying $\lim _{n \rightarrow \infty} \varepsilon_{n}=0, \lim _{n \rightarrow \infty}\left\|u_{n}-u\right\|_{L_{\mu}^{p}\left(X ; \mathbb{R}^{m}\right)}=0$ and $\sup _{n \in \mathbb{N}}\left\|\nabla_{\mu} u_{n}\right\|_{L_{\mu}^{p}(X ; \mathbb{M})}^{p}<\infty$ where $u_{n}:=u_{\varepsilon_{n}}$ for all $n \in \mathbb{N}$. Without loss of generality we can assume, up to a subsequence (not relabelled), that

$$
\begin{array}{ll} 
& \sup _{n \in \mathbb{N}} I\left(u_{n} ; O\right)<\infty, \varliminf_{n \rightarrow \infty} I\left(u_{n} ; O\right)<\infty, \sup _{n \in \mathbb{N}} \int_{O}\left|\nabla_{\mu} u_{n}\right|^{p} d \mu<\infty \\
\text { and } & \lim _{n \rightarrow \infty}\left\|u_{n}-u\right\|_{L_{\mu}^{p}\left(X ; \mathbb{R}^{m}\right)}=0 .
\end{array}
$$

For each $n \in \mathbb{N}$ we set

$$
\nu_{n}:=L\left(\cdot, \nabla_{\mu} u_{n}(\cdot)\right) \mu \iota_{O} \quad \text { and } \quad \lambda_{n}:=\left|\nabla_{\mu} u_{n}\right|^{p} \mu\left\lfloor_{O}\right.
$$

which are nonnegative Borel regular measures on $O$. Apply $\left(\mathrm{AL}_{X}\right)$ by using the boundedness conditions in (4.3), there exist subsequences (not relabelled) $\left\{\nu_{n}\right\}_{n \in \mathbb{N}},\left\{\lambda_{n}\right\}_{n \in \mathbb{N}}$ and locally finite Borel regular measures $\nu, \lambda$ such that

$$
\begin{aligned}
& \varliminf_{n \rightarrow \infty} \nu_{n}(V) \geqslant \nu(V) \text { and } \underline{\lim }_{n \rightarrow \infty} \lambda_{n}(V) \geqslant \lambda(V) \quad \text { for all open set } V \subset O \\
& \varlimsup_{n \rightarrow \infty} \nu_{n}(B) \leqslant \nu(B) \text { and } \varlimsup_{n \rightarrow \infty} \lambda_{n}(B) \leqslant \lambda(B) \quad \text { for all closed ball } B \subset O .
\end{aligned}
$$

Step1: localization. By the Lebesgue decomposition theorem and the Radon-Nikodym theorem there exist $\nu^{s}$, which is mutually singular with respect to $\left.\mu\right|_{O}$, and $\Phi \in L_{\mu}^{1}(O)$ such that

$$
\nu=\Phi \mu \iota_{O}+\nu^{s} \quad \text { and } \quad \Phi(x)=\lim _{\rho \rightarrow 0} \frac{\nu\left(B_{\rho}(x)\right)}{\mu\left(B_{\rho}(x)\right)} \quad \mu \text {-a.e. in } O .
$$

Similarly, there exist $\lambda^{s}$, which is mutually singular with respect to $\mu$, and $\frac{d \lambda}{d \mu} \in L_{\mu}^{1}(O)$ such that

$$
\lambda=\frac{d \lambda}{d \mu} \mu\left\lfloor_{O}+\lambda^{s} \quad \text { and } \quad \frac{d \lambda}{d \mu}(x)=\lim _{\rho \rightarrow 0} \frac{\lambda\left(B_{\rho}(x)\right)}{\mu\left(B_{\rho}(x)\right)} \quad \mu \text {-a.e. in } O\right. \text {. }
$$

(Note that because of the doubling property of $\mu$, we can replace closed balls by open balls in the differentiation formula above, see [HKST15, Remark 3.4.29, pp. 86].) 
Let $t \in] 0,1\left[\right.$. By (4.5), (4.6) and the annular decay property $\left(\mathrm{AD}_{X}\right)$, we have for $\mu$-a.e. $x \in O$

$$
\begin{aligned}
\Phi(x) \geqslant \varlimsup_{\rho \rightarrow 0} \varlimsup_{n \rightarrow \infty} \frac{\nu_{n}\left(\bar{B}_{t \rho}(x)\right)}{\mu\left(B_{\rho}(x)\right)} & \geqslant \varlimsup_{\rho \rightarrow 0} \varlimsup_{n \rightarrow \infty} \frac{\nu_{n}\left(B_{t \rho}(x)\right)}{\mu\left(B_{t \rho}(x)\right)} \frac{\mu\left(B_{t \rho}(x)\right)}{\mu\left(B_{\rho}(x)\right)} \\
& \geqslant\left(1-K(1-t)^{\delta}\right) \varlimsup_{\rho \rightarrow 0} \varlimsup_{n \rightarrow \infty} \frac{\nu_{n}\left(B_{t \rho}(x)\right)}{\mu\left(B_{t \rho}(x)\right)},
\end{aligned}
$$

letting $t \rightarrow 1$ we have

$$
\Phi(x) \geqslant \varlimsup_{t \rightarrow 1} \varlimsup_{\rho \rightarrow 0} \varlimsup_{n \rightarrow \infty} f_{B_{t \rho}(x)} L\left(y, u_{n}, \nabla_{\mu} u_{n}\right) d \mu .
$$

Let $k \in \mathbb{N}$ and fix $x \in O \cap X_{k}$, where $\left(X_{k}, \gamma^{k}\right)$ is given by Theorem 1 , satisfying

$$
\begin{aligned}
\mathbb{R}^{m} \times \mathrm{M} \ni(v, \xi) & \longmapsto L(x, v, \xi) \text { is lower semicontinuous; } \\
\infty>\Phi(x) & =\lim _{\rho \rightarrow 0} \frac{\nu\left(B_{\rho}(x)\right)}{\mu\left(B_{\rho}(x)\right)} ; \\
\Phi(x) & \geqslant \varlimsup_{t \rightarrow 1} \varlimsup_{\rho \rightarrow 0} \varlimsup_{n \rightarrow \infty} f_{B_{t \rho}(x)} L\left(y, u_{n}, \nabla_{\mu} u_{n}\right) d \mu ; \\
\infty>A(x) & =\lim _{\rho \rightarrow 0} f_{B_{\rho}(x)} A(y) d \mu(y) ; \\
\infty>|u(x)|^{p} & =\lim _{\rho \rightarrow 0} f_{B_{\rho}(x)}|u(y)|^{p} d \mu(y) ; \\
\infty>\frac{d \lambda}{d \mu}(x) & :=\lim _{\rho \rightarrow 0} \frac{\lambda\left(B_{\rho}(x)\right)}{\mu\left(B_{\rho}(x)\right)} ; \\
\infty & >\left|\nabla_{\mu} u(x)\right|^{p} ; \\
0 & =\lim _{\rho \rightarrow 0} \frac{1}{\rho^{p}} f_{B_{\rho}(x)}\left|u(y)-u_{x}(y)\right|^{p} d \mu(y) .
\end{aligned}
$$

The equalities and inequalities above are fullfilled $\mu$-a.e. in $X$ because of the assumptions of Theorem 5 and Proposition 1 (iv).

For each $\delta>0$, there exist $v_{\delta}^{x} \in \mathbb{Q}^{m}$ and $F_{\delta}^{x} \in \mathbb{Q}^{m N}$ (that is with rational numbers entries) such that $\left|v_{\delta}^{x}-u(x)\right|<\delta$ and $\left|F_{\delta}^{x}-\nabla_{\mu} u(x)\right|<\delta$. We set $u_{x, \delta}(\cdot):=\Lambda_{x, v_{\delta}^{x}, F_{\delta}^{x}}^{k}(\cdot)=$ $v_{\delta}^{x}+F_{\delta}^{x} \cdot\left(\gamma^{k}(\cdot)-\gamma^{k}(x)\right)$. By Remark2 we have

$$
L\left(x, v_{\delta}^{x}, F_{\delta}^{x}\right) \leqslant \underline{\lim _{\rho \rightarrow 0}} \inf _{\varphi \in H_{\mu, 0}^{1, p}\left(B_{\rho}(x) ; \mathbb{R}^{m}\right)} f_{B_{\rho}(x)} L\left(y, u_{x, \delta}+\varphi, F_{\delta}^{x}+\nabla_{\mu} \varphi\right) d \mu .
$$

Step 2: cut-off technique. Fix $\rho \in] 0,1\left[\right.$ satisfying $B_{\rho}(x) \subset O$. By Proposition 1 (v), we consider an Urysohn function $\varphi \in \operatorname{Lip}_{0}\left(B_{\rho}(x)\right)$ for the pair $\left(X \backslash B_{t \rho}(x), \bar{B}_{t^{2} \rho}(x)\right)$ which means that $\varphi \in \operatorname{Lip}(X ;[0,1])$ with $\varphi \equiv 0$ on $X \backslash B_{t \rho}(x)$ and $\varphi \equiv 1$ on $\bar{B}_{t^{2} \rho}(x)$ (note that $t^{2}<t$ since $\left.t \in\right] 0,1[)$. Moreover, there exists $C_{0}>0$ such that $\left\|D_{\mu} \varphi\right\|_{L_{\mu}^{\infty}\left(X ; \mathbb{R}^{N}\right)} \leqslant \frac{C_{0}}{t(1-t) \rho}$.

Fix $n \in \mathbb{N}$ and $\varepsilon \in] 0,1[$. We set $\delta=\delta(\varepsilon, t, \rho):=t(1-t) \rho \varepsilon$ and

$$
\widetilde{u}:=\varphi u_{n}+(1-\varphi) u_{x, \delta}=\varphi\left(u_{n}-u_{x, \delta}\right)+u_{x, \delta}
$$


where $u_{x, \delta}(\cdot)=\Lambda_{x, v_{\delta}^{x}, F_{\delta}^{x}}^{k}(\cdot)=v_{\delta(\varepsilon, t, \rho)}^{x}+F_{\delta(\varepsilon, t, \rho)}^{x} \cdot\left(\gamma^{k}(\cdot)-\gamma^{k}(x)\right)$, and which satisfies for $\mu$-a.e. $y \in B_{\rho}(x)$

$$
\nabla_{\mu} \widetilde{u}=\left\{\begin{array}{cl}
\nabla_{\mu} u_{n} & \text { on } \bar{B}_{t^{2} \rho}(x) \\
\varphi \nabla_{\mu} u_{n}+(1-\varphi) F_{\delta}^{x}+D_{\mu} \varphi \otimes\left(u_{n}-u_{x, \delta}\right) & \text { on } B_{t \rho}(x) \backslash \bar{B}_{t^{2} \rho}(x) \\
F_{\delta}^{x} & \text { on } B_{\rho}(x) \backslash B_{t \rho}(x) .
\end{array}\right.
$$

We see that $\widetilde{u} \in u_{x, \delta}+H_{\mu, 0}^{1, p}\left(B_{\rho}(x) ; \mathbb{R}^{m}\right)$, and we have

$$
\frac{I\left(\widetilde{u} ; B_{\rho}(x)\right)}{\mu\left(B_{\rho}(x)\right)} \leqslant \frac{I\left(u_{n} ; B_{t \rho}(x)\right)}{\mu\left(B_{t \rho}(x)\right)}+\frac{1}{\mu\left(B_{\rho}(x)\right)}\left(I\left(\widetilde{u} ; B_{t \rho}(x) \backslash \bar{B}_{t^{2} \rho}(x)\right)+I\left(u_{x, \delta} ; B_{\rho}(x) \backslash B_{t \rho}(x)\right)\right) .
$$

We estimate the last two terms on the right hand side of (4.17) by using the growth condition 4.2. We have

$$
\begin{aligned}
& I\left(\tilde{u} ; B_{t \rho}(x) \backslash \bar{B}_{t^{2} \rho}(x)\right)+I\left(u_{x, \delta} ; B_{\rho}(x) \backslash B_{t \rho}(x)\right) \\
\leqslant & \int_{B_{t \rho}(x) \backslash \bar{B}_{t^{2} \rho}(x)} A(y)+C\left(1+|\widetilde{u}|^{p}+\left|\nabla_{\mu} \widetilde{u}\right|^{p}\right) d \mu \\
& +\int_{B_{\rho}(x) \backslash B_{t \rho}(x)} A(y)+C\left(1+\left|u_{x, \delta}\right|^{p}+\left|F_{\delta}^{x}\right|^{p}\right) d \mu \\
\leqslant & \int_{B_{\rho}(x) \backslash \bar{B}_{t^{2} \rho}(x)}(A(y)+C) d \mu+C 2^{p-1} \int_{B_{t \rho}(x) \backslash \bar{B}_{t^{2} \rho}(x)}\left|u_{n}\right|^{p} d \mu \\
& +C\left(2^{p-1}+1\right) \int_{B_{\rho}(x) \backslash B_{t \rho}(x)}\left|u_{x, \delta}\right|^{p} d \mu+C\left|F_{\delta}^{x}\right|^{p} \mu\left(B_{\rho}(x) \backslash B_{t \rho}(x)\right) \\
& +C 2^{2 p-2} \int_{B_{t \rho}(x) \backslash \bar{B}_{t^{2} \rho}(x)}\left|\nabla_{\mu} u_{n}\right|^{p}+\left|F_{\delta}^{x}\right|^{p}+|| D_{\mu} \varphi \|_{L_{\mu}^{\infty}\left(X ; \mathbb{R}^{N}\right)}^{p}\left|u_{n}-u_{x, \delta}\right|^{p} d \mu \\
\leqslant & \int_{B_{\rho}(x) \backslash \bar{B}_{t^{2} \rho}(x)}(A(y)+C) d \mu+C\left(2^{2 p-2}+1\right)\left|F_{\delta}^{x}\right|^{p} \mu\left(B_{\rho}(x) \backslash B_{t^{2} \rho}(x)\right) \\
& +C 2^{2 p-2}\left(\int_{B_{t \rho}(x) \backslash \bar{B}_{t^{2} \rho}(x)}\left|u_{n}-u\right|^{p} d \mu+\int_{B_{\rho}(x) \backslash \bar{B}_{t^{2} \rho}(x)}|u|^{p} d \mu\right. \\
& \left.+\int_{B_{\rho}(x) \backslash B_{t \rho}(x)}\left|u_{x, \delta}-u\right|^{p} d \mu\right) \\
& +C 2^{2 p-2} \int_{B_{t \rho}(x) \backslash \bar{B}_{t^{2} \rho}(x)}\left|\nabla_{\mu} u_{n}\right|^{p}+\frac{C_{0}^{p}}{t^{p}(1-t)^{p} \rho^{p}}\left(\left|u_{n}-u\right|^{p}+\left|u-u_{x, \delta}\right|^{p}\right) d \mu .
\end{aligned}
$$


Set $C^{\prime}:=C\left(2^{2 p-2}+1\right)$. Using the estimate above in (4.17), we have

$$
\begin{aligned}
\inf _{\varphi \in H_{\mu, 0}^{1, p}\left(B_{\rho}(x) ; \mathbb{R}^{m}\right)} & f_{B_{\rho}(x)} L\left(y, u_{x, \delta}+\varphi, F_{\delta}^{x}+\nabla_{\mu} \varphi\right) d \mu \\
\leqslant & \frac{I\left(\tilde{u} ; B_{\rho}(x)\right)}{\mu\left(B_{\rho}(x)\right)} \\
\leqslant & \frac{I\left(u_{n} ; B_{t \rho}(x)\right)}{\mu\left(B_{t \rho}(x)\right)}+\frac{1}{\mu\left(B_{\rho}(x)\right)} \int_{B_{\rho}(x) \backslash \bar{B}_{t^{2} \rho}(x)}(A(y)+C) d \mu \\
& +C^{\prime}\left|F_{\delta}^{x}\right|^{p} \frac{\mu\left(B_{\rho}(x) \backslash B_{t^{2} \rho}(x)\right)}{\mu\left(B_{\rho}(x)\right)}+\frac{C^{\prime}}{\mu\left(B_{\rho}(x)\right)} \int_{B_{t \rho}(x) \backslash \bar{B}_{t^{2} \rho}(x)}\left|\nabla_{\mu} u_{n}\right|^{p} d \mu \\
& +\frac{C^{\prime}}{\mu\left(B_{\rho}(x)\right)}\left(\int_{B_{\rho}(x)}\left|u_{n}-u\right|^{p} d \mu+\int_{B_{\rho}(x) \backslash \bar{B}_{t^{2} \rho}(x)}|u|^{p} d \mu\right. \\
& +\int_{B_{\rho}(x) \backslash B_{t \rho}(x)}^{\left.\left|u_{x, \delta}-u\right|^{p} d \mu\right)} \\
& +C^{\prime} f_{B_{\rho}(x)} \frac{C_{0}^{p}}{t^{p}(1-t)^{p} \rho^{p}}\left(\left|u_{n}-u\right|^{p}+\left|u-u_{x, \delta}\right|^{p}\right) d \mu .
\end{aligned}
$$

Step 3: estimates and passing to the limit. We set

$$
\begin{aligned}
\Delta_{x, t, \rho}^{1} & :=\frac{1}{\mu\left(B_{\rho}(x)\right)} \int_{B_{\rho}(x) \backslash \bar{B}_{t^{2} \rho}(x)}(A(y)+C) d \mu \\
\Delta_{x, t, \rho}^{2} & :=C^{\prime}\left|F_{\delta}^{x}\right|^{p} \frac{\mu\left(B_{\rho}(x) \backslash B_{t^{2} \rho}(x)\right)}{\mu\left(B_{\rho}(x)\right)} \\
\Delta_{x, t, \rho, n}^{3} & :=\frac{C^{\prime}}{\mu\left(B_{\rho}(x)\right)} \int_{B_{t \rho}(x) \backslash \bar{B}_{t^{2} \rho}(x)}\left|\nabla_{\mu} u_{n}\right|^{p} d \mu \\
\Delta_{x, t, \rho, n}^{4} & :=\left(\frac{C^{\prime} C_{0}^{p}}{t^{p}(1-t)^{p} \rho^{p}}+C^{\prime}\right) f_{B_{\rho}(x)}\left|u_{n}-u\right|^{p} d \mu \\
\Delta_{x, t, \rho}^{5} & :=\left(\frac{C^{\prime} C_{0}^{p}}{t^{p}(1-t)^{p}}+\rho^{p} C^{\prime}\right) \frac{1}{\rho^{p}} f_{B_{\rho}(x)}\left|u-u_{x, \delta}\right|^{p} d \mu \\
\Delta_{x, t, \rho, \varepsilon}^{6} & :=\frac{C^{\prime}}{\mu\left(B_{\rho}(x)\right)} \int_{B_{\rho}(x) \backslash \bar{B}_{t^{2} \rho}(x)}|u|^{p} d \mu
\end{aligned}
$$

Since $\lim _{n \rightarrow \infty}\left\|u_{n}-u\right\|_{L_{\mu}^{p}\left(X ; \mathbb{R}^{m}\right)}=0$ in $L_{\mu}^{p}\left(X ; \mathbb{R}^{m}\right)$ we have

$$
\lim _{n \rightarrow \infty} \Delta_{x, t, \rho, n}^{4}=0 \text {. }
$$

Set $C^{\prime \prime}:=2^{p} C^{\prime} \max \left(1, C_{0}^{p}\right)$. We have

$$
\begin{aligned}
& \Delta_{x, t, \rho, \varepsilon}^{5} \\
& \leqslant C^{\prime \prime}\left((t(1-t))^{-p}+\rho^{p}\right)\left(\frac{1}{\rho^{p}} f_{B_{\rho}(x)}\left|u-u_{x}\right|^{p} d \mu+\frac{1}{\rho^{p}} f_{B_{\rho}(x)}\left|u_{x}-u_{x, \delta}\right|^{p} d \mu\right) \\
& \leqslant 2^{p} C^{\prime \prime}\left((t(1-t))^{-p}+1\right)\left(\frac{1}{\rho^{p}} f_{B_{\rho}(x)}\left|u-u_{x}\right|^{p} d \mu+\frac{1}{\rho^{p}} f_{B_{\rho}(x)}(t(1-t) \rho)^{p} \varepsilon^{p}\left(1+C_{k}^{p} \rho^{p}\right) d \mu\right),
\end{aligned}
$$


where $C_{k}$ is the Lipschitz constant of the Lipschitz function $\gamma^{k}$, i.e.

$$
\left|\gamma^{k}(y)-\gamma^{k}(z)\right| \leqslant C_{k} d(z, y) \text { for all }(y, z) \in X^{2} .
$$

On one hand, using 4.15 we have

$$
\lim _{\rho \rightarrow 0} 2^{p} C^{\prime \prime}\left((t(1-t))^{-p}+1\right) \frac{1}{\rho^{p}} f_{B_{\rho}(x)}\left|u-u_{x}\right|^{p} d \mu=0 .
$$

On the other hand, if we set $C^{\prime \prime \prime}:=2^{p+1} C^{\prime \prime}\left(1+C_{k}^{p}\right)$ we have

$$
2^{p} C^{\prime \prime}\left((t(1-t))^{-p}+1\right) \frac{1}{\rho^{p}} f_{B_{\rho}(x)}(t(1-t) \rho)^{p} \varepsilon^{p}\left(1+C_{k}^{p} \rho^{p}\right) d \mu \leqslant C^{\prime \prime \prime} \varepsilon^{p} .
$$

It follows that

$$
\varlimsup_{\varepsilon \rightarrow 0} \varlimsup_{t \rightarrow 1} \varlimsup_{\rho \rightarrow 0} \Delta_{x, t, \rho, \varepsilon}^{5}=0 .
$$

By the annular decay property $\left(\mathrm{AD}_{X}\right)$ and 4.14 we have $\Delta_{x, t, \rho}^{2} \leqslant C^{\prime}\left|F_{\delta}^{x}\right|^{p} K\left(1-t^{2}\right)^{\eta}$, so letting $\rho \rightarrow 0$ and $t \rightarrow 1$ we obtain

$$
\lim _{t \rightarrow 1} \varlimsup_{\rho \rightarrow 0} \Delta_{x, t, \rho}^{2}=0 .
$$

We have

$$
\frac{1}{C^{\prime}} \Delta_{x, t, \rho, n}^{3}=\frac{1}{\mu\left(B_{\rho}(x)\right)}\left(\lambda_{n}\left(B_{t \rho}(x)\right)-\lambda_{n}\left(\bar{B}_{t^{2} \rho}(x)\right)\right) \leqslant \frac{\lambda_{n}\left(\bar{B}_{t \rho}(x)\right)}{\mu\left(B_{\rho}(x)\right)}-\frac{\lambda_{n}\left(B_{t^{2} \rho}(x)\right)}{\mu\left(B_{\rho}(x)\right)} .
$$

Passing to limit $n \rightarrow \infty$ and using (4.5), we have by the annular decay property

$$
\begin{aligned}
\varlimsup_{n \rightarrow \infty} \frac{1}{C^{\prime}} \Delta_{x, t, \rho, n}^{3} & \leqslant \frac{\lambda\left(\bar{B}_{t \rho}(x)\right)}{\mu\left(B_{\rho}(x)\right)}-\frac{\lambda\left(B_{t^{2} \rho}(x)\right)}{\mu\left(B_{\rho}(x)\right)} \\
& =\frac{\lambda\left(\bar{B}_{t \rho}(x)\right)}{\mu\left(\bar{B}_{t \rho}(x)\right)} \frac{\mu\left(\bar{B}_{t \rho}(x)\right)}{\mu\left(B_{\rho}(x)\right)}-\frac{\lambda\left(B_{t^{2} \rho}(x)\right)}{\mu\left(B_{t^{2} \rho}(x)\right)} \frac{\mu\left(B_{t^{2} \rho}(x)\right)}{\mu\left(B_{\rho}(x)\right)} \\
& \leqslant \frac{\lambda\left(\bar{B}_{t \rho}(x)\right)}{\mu\left(\bar{B}_{t \rho}(x)\right)}-\frac{\lambda\left(B_{t^{2} \rho}(x)\right)}{\mu\left(B_{t^{2} \rho}(x)\right)} \frac{\mu\left(B_{t^{2} \rho}(x)\right)}{\mu\left(B_{\rho}(x)\right)} \\
& =\frac{\lambda\left(\bar{B}_{t \rho}(x)\right)}{\mu\left(\bar{B}_{t \rho}(x)\right)}+\frac{\lambda\left(B_{t^{2} \rho}(x)\right)}{\mu\left(B_{t^{2} \rho}(x)\right)}\left(\frac{\mu\left(B_{\rho}(x) \backslash B_{t^{2} \rho}(x)\right)}{\mu\left(B_{\rho}(x)\right)}-1\right) \\
& \leqslant \frac{\lambda\left(\bar{B}_{t \rho}(x)\right)}{\mu\left(\bar{B}_{t \rho}(x)\right)}+\frac{\lambda\left(B_{t^{2} \rho}(x)\right)}{\mu\left(B_{t^{2} \rho}(x)\right)}\left(K\left(1-t^{2}\right)^{\eta}-1\right) .
\end{aligned}
$$

Now, on taking account of (4.13) we have

$$
\varlimsup_{\rho \rightarrow 0} \varlimsup_{n \rightarrow \infty} \Delta_{x, t, \rho, n}^{3} \leqslant C^{\prime} K\left(1-t^{2}\right)^{\eta} \frac{d \lambda}{d \mu}(x)
$$

it follows by letting $t \rightarrow 1$ that

$$
\varlimsup_{t \rightarrow 1} \varlimsup_{\rho \rightarrow 0} \varlimsup_{n \rightarrow \infty} \Delta_{x, t, \rho, n}^{3}=0 .
$$


We have

$$
\begin{aligned}
\Delta_{x, t, \rho}^{1} & =f_{B_{\rho}(x)} A(y) d \mu(y)-\frac{\mu\left(B_{t^{2} \rho}(x)\right)}{\mu\left(B_{\rho}(x)\right)} f_{B_{t^{2} \rho}(x)} A(y) d \mu(y)+C \frac{\mu\left(B_{\rho}(x) \backslash B_{t^{2} \rho}(x)\right)}{\mu\left(B_{\rho}(x)\right)} \\
& =f_{B_{\rho}(x)} A(y) d \mu(y)+\left(\frac{\mu\left(B_{\rho}(x) \backslash B_{t^{2} \rho}(x)\right)}{\mu\left(B_{\rho}(x)\right)}-1\right) f_{B_{t^{2} \rho}(x)} A(y) d \mu(y) \\
& +C \frac{\mu\left(B_{\rho}(x) \backslash B_{t^{2} \rho}(x)\right)}{\mu\left(B_{\rho}(x)\right)} \\
& \leqslant f_{B_{\rho}(x)} A(y) d \mu(y)+\left(K\left(1-t^{2}\right)^{\eta}-1\right) f_{B_{t^{2} \rho}(x)} A(y) d \mu(y)+C\left(K\left(1-t^{2}\right)^{\eta}\right) .
\end{aligned}
$$

Taking (4.11) into account and passing to the limit $\rho \rightarrow 0$ we have

$$
\varlimsup_{\rho \rightarrow 0} \Delta_{x, t, \rho}^{1} \leqslant(A(x)+C)\left(K\left(1-t^{2}\right)^{\eta}\right),
$$

and letting $t \rightarrow 1$ we obtain

$$
\lim _{t \rightarrow 1} \varlimsup_{\rho \rightarrow 0} \Delta_{x, t, \rho}^{1}=0 .
$$

For $\Delta_{x, t, \rho}^{6}$ we proceed similarly, i.e.

$$
\begin{aligned}
\frac{1}{C^{\prime}} \Delta_{x, t, \rho}^{6} & =f_{B_{\rho}(x)}|u|^{p} d \mu-\frac{\mu\left(B_{t^{2} \rho}(x)\right)}{\mu\left(B_{\rho}(x)\right)} f_{B_{t^{2} \rho}(x)}|u|^{p} d \mu \\
& =f_{B_{\rho}(x)}|u|^{p} d \mu+\left(\frac{\mu\left(B_{\rho}(x) \backslash B_{t^{2} \rho}(x)\right)}{\mu\left(B_{\rho}(x)\right)}-1\right) f_{B_{t^{2} \rho}(x)}|u|^{p} d \mu \\
& \leqslant f_{B_{\rho}(x)}|u|^{p} d \mu+\left(K\left(1-t^{2}\right)^{\eta}-1\right) f_{B_{t^{2} \rho}(x)}|u|^{p} d \mu .
\end{aligned}
$$

Using (4.12) and letting $\rho \rightarrow 0$ we have

$$
\varlimsup_{\rho \rightarrow 0} \Delta_{x, t, \rho}^{6} \leqslant K\left(1-t^{2}\right)^{\eta} C^{\prime}|u(x)|^{p},
$$

and by passing to the limit $t \rightarrow 1$ we obtain

$$
\varlimsup_{t \rightarrow 1} \varlimsup_{\rho \rightarrow 0} \Delta_{x, t, \rho}^{6}=0 .
$$

Step 4: end of the proof. Collecting 4.18), 4.19, 4.20), 4.21, 4.22) and 4.23) we have by (4.8), 4.16) and (4.10)

$$
\begin{aligned}
L\left(x, u(x), \nabla_{\mu} u(x)\right) & \leqslant \varlimsup_{\varepsilon \rightarrow 0} \varlimsup_{t \rightarrow 1} \varlimsup_{\rho \rightarrow 0} L\left(x, v_{\delta}^{x}, F_{\delta}^{x}\right) \\
& \leqslant \varlimsup_{\varepsilon \rightarrow 0} \varlimsup_{t \rightarrow 1} \varlimsup_{\rho \rightarrow 0} \inf _{\varphi \in H_{\mu, 0}^{1, p}\left(B_{\rho}(x) ; \mathbb{R}^{m}\right)} f_{B_{\rho}(x)} L\left(y, u_{x, \delta}+\varphi, F_{\delta}^{x}+\nabla_{\mu} \varphi\right) d \mu \\
& \leqslant \varlimsup_{t \rightarrow 1} \varlimsup_{\rho \rightarrow 0} \varlimsup_{n \rightarrow \infty} f_{B_{t \rho}(x)} L\left(y, u_{n}, \nabla_{\mu} u_{n}\right) d \mu \leqslant \Phi(x) .
\end{aligned}
$$

Now, to finish the proof we use 4.4 and 4.6

$$
\begin{aligned}
I(u ; O) & =\int_{O} L\left(x, u(x), \nabla_{\mu} u(x)\right) d \mu(x) \\
& \leqslant \int_{O} \Phi(x) d \mu(x) \leqslant \nu(O) \leqslant \varliminf_{n \rightarrow \infty} \nu_{n}(O)=\varliminf_{n \rightarrow \infty} I\left(u_{n} ; O\right) .
\end{aligned}
$$


4.2. Necessary and sufficient condition for lower semicontinuity. Consider the integral functional $I: H_{\mu}^{1, p}\left(X ; \mathbb{R}^{m}\right) \times \mathcal{O}(X) \longrightarrow[0, \infty]$ associated with a Borel measurable integrand $L: X \times \mathbb{M} \longrightarrow[0, \infty]$, defined by

$$
I(u ; O):=\int_{O} L\left(x, \nabla_{\mu} u\right) d \mu .
$$

When $X=O$ we write $I(u)$ in place of $I(u ; X)$ for all $u \in H_{\mu}^{1, p}\left(X ; \mathbb{R}^{m}\right)$.

If $\mu$ is finite and $L$ is $p$-coercive and has $p$-polynomial growth, by using Theorem 5 , Theorem 2 and Lemma 2, we have the following characterization of the lower semicontinuity of $I$.

Theorem 6. Assume that $(X, d, \mu)$ satisfies $\left(\mathrm{AD}_{X}\right)$, $\left(\mathrm{AL}_{X}\right)$ and $\mu$ is finite.

Let $L: X \times \mathbb{M} \longrightarrow[0, \infty]$ be a Borel measurable (resp. Carathéodory) integrand such that for $\mu$-a.e. $x \in X$ the function $L(x, \cdot)$ is lower semicontinuous. Assume that there exist $c, C>0$ and $A \in L_{\mu}^{1}(X)$ such that for $\mu$-a.e. $x \in X$

$$
c|\xi|^{p} \leqslant L(x, \xi) \leqslant A(x)+C\left(1+|\xi|^{p}\right) \quad \text { for all } \xi \in \mathbb{M} .
$$

Then $L$ is $H_{\mu}^{1, p}$-quasiconvex (resp. Lip-quasiconvex) if and only if $I(\cdot)$ is lower semicontinuous with respect to the strong convergence of $L_{\mu}^{p}\left(X ; \mathbb{R}^{m}\right)$. have:

In case $\mu$ not necessarily finite, by using Theorem 5, Theorem 3 and Lemma 2, we

Theorem 7. Assume that $(X, d, \mu)$ satisfies $\left(\mathrm{AD}_{X}\right)$ and $\left(\mathrm{AL}_{X}\right)$,

Let $L: X \times \mathbb{M} \longrightarrow[0, \infty]$ be a Borel measurable (resp. Carathéodory) integrand such that for $\mu$-a.e. $x \in X$ the function $L(x, \cdot)$ is lower semicontinuous. Assume that there exist c, $C>0$ and $A \in L_{\mu, \mathrm{loc} *}^{1}(X)$ such that for $\mu$-a.e. $x \in X$

$$
c|\xi|^{p} \leqslant L(x, \xi) \leqslant A(x)+C\left(1+|\xi|^{p}\right) \quad \text { for all } \xi \in \mathbb{M} .
$$

Then $L$ is $H_{\mu}^{1, p}$-quasiconvex (resp. Lip-quasiconvex) if and only if $I(\cdot ; O)$ is lower semicontinuous with respect to the strong convergence of $L_{\mu}^{p}\left(X ; \mathbb{R}^{m}\right)$ for all $O \in \mathcal{O}(X)$ with $\mu(O)<\infty$.

\section{A Class of NONCONVEX Integrands Without $p$-GROWTH}

In the spirit of the class of polyconvex integrands, see for instance [BM84, we provide a class of $H_{\mu}^{1, p}$-quasiconvex integrands which are nonconvex and that may not have polynomial growth. These are composition of convex functions and $H_{\mu}^{1, p}$-quasiconvex integrands. In the Euclidean setting with the Lebesgue measure $(X, d, \mu)=\left(\Omega,|\cdot-\cdot|, \mathcal{L}_{N} \mid \Omega\right)$, it is direct to see that if $g(\cdot)$ is convex and nondecreasing with respect to each of its variables then the composite integrand $L(\cdot)=g\left(f_{1}(\cdot), \cdots, f_{i}(\cdot), \cdots, f_{s}(\cdot)\right)$ with each $f_{i}(\cdot)$ quasiconvex, is quasiconvex because of the Jensen inequality. In the metric measure space setting, we can also use the Jensen inequality to have a similar result.

Proposition 3. Let $g: \mathbb{R}^{s} \rightarrow[0, \infty]$ be a convex and lower semicontinuous function which is nondecreasing with respect to each of its variables, i.e. for every $i \in\{1, \cdots, s\}$ and every $t_{j} \in \mathbb{R}$ with $j \neq i$ the function

$$
\mathbb{R} \ni t_{i} \longmapsto g\left(t_{1}, \cdots, t_{i}, \cdots, t_{s}\right) \text { is nondecreasing. }
$$

Let $\left\{f_{i}\right\}_{i=1}^{s}, f_{i}: X \times \mathbb{M} \longrightarrow[0, \infty]$ be a finite family of $H_{\mu}^{1, p}$-quasiconvex integrands. The composite integrand $L=g \circ F$ where $F(x, \xi)=\left(f_{1}(x, \xi), \cdots, f_{i}(x, \xi), \cdots, f_{s}(x, \xi)\right)$ for all $(x, \xi) \in X \times \mathbb{M}$ is $H_{\mu}^{1, p}$-quasiconvex. 
Proof. Let $\xi \in \mathbb{M}$. By Jensen inequality and the properties of $g$, we have for $\mu$-a.e. $x \in X$

$$
\begin{aligned}
& \varliminf_{\rho \rightarrow 0} \inf _{\varphi \in H_{\mu, 0}^{1, p}\left(B_{\rho}(x) ; \mathbb{R}^{m}\right)} f_{\bar{B}_{\rho}(x)} L\left(y, \xi+\nabla_{\mu} \varphi\right) d \mu \\
& \geqslant \varliminf_{\rho \rightarrow 0} \inf _{\varphi \in H_{\mu, 0}^{1, p}\left(B_{\rho}(x) ; \mathbb{R}^{m}\right)} g\left(f_{\bar{B}_{\rho}(x)} f_{1}\left(y, \xi+\nabla_{\mu} \varphi\right) d \mu, \cdots, f_{\bar{B}_{\rho}(x)} f_{s}\left(y, \xi+\nabla_{\mu} \varphi\right) d \mu\right) \\
& \geqslant \lim _{\rho \rightarrow 0} g\left(\inf _{\varphi \in H_{\mu, 0}^{1, p}\left(B_{\rho}(x) ; \mathbb{R}^{m}\right)} f_{\bar{B}_{\rho}(x)} f_{1}\left(y, \xi+\nabla_{\mu} \varphi\right) d \mu\right. \\
& \left., \cdots, \inf _{\varphi \in H_{\mu, 0}^{1, p}\left(B_{\rho}(x) ; \mathbb{R}^{m}\right)} f_{\bar{B}_{\rho}(x)} f_{s}\left(y, \xi+\nabla_{\mu} \varphi\right) d \mu\right) \\
& \geqslant g\left(\lim _{\rho \rightarrow 0} \inf _{\varphi \in H_{\mu, 0}^{1, p}\left(B_{\rho}(x) ; \mathbb{R}^{m}\right)} f_{\bar{B}_{\rho}(x)} f_{1}\left(y, \xi+\nabla_{\mu} \varphi\right) d \mu\right. \\
& \left., \cdots, \lim _{\rho \rightarrow 0} \inf _{\varphi \in H_{\mu, 0}^{1, p}\left(B_{\rho}(x) ; \mathbb{R}^{m}\right)} f_{\bar{B}_{\rho}(x)} f_{s}\left(y, \xi+\nabla_{\mu} \varphi\right) d \mu\right) \\
& \geqslant g(F(x, \xi))=L(x, \xi) .
\end{aligned}
$$

In Corollary 2 below we extend Proposition 3 for convex function $g$ depending on $(x, \xi)$. To achieve this task we first show a lower semicontinuous result for such $g$.

Lemma 3. Assume that $(X, d, \mu)$ satisfies $\left(\mathrm{AD}_{X}\right)$ and $\left(\mathrm{AL}_{X}\right)$. Let $L: X \times \mathbb{M} \longrightarrow[0, \infty]$ be a $H_{\mu}^{1, p}$-quasiconvex Carathéodory integrand. Assume that $L$ has q-polynomial growth with $q \in] 1, p\left[\right.$, i.e. there exist $C>0$ and $A \in L_{\mu, \text { loc }}^{1}(X)$ such that for $\mu$-a.e. $x \in X$

$$
L(x, \xi) \leqslant A(x)+C\left(1+|\xi|^{q}\right) \quad \text { for all } \xi \in \mathbb{M} .
$$

Then for every $u,\left\{u_{\varepsilon}\right\}_{\varepsilon>0} \subset H_{\mu}^{1, p}\left(X ; \mathbb{R}^{m}\right)$ satisfying

$$
\lim _{\varepsilon \rightarrow 0}\left\|u_{\varepsilon}-u\right\|_{L_{\mu}^{p}\left(X ; \mathbb{R}^{m}\right)}=0 \text { and } \sup _{\varepsilon>0}\left\|\nabla_{\mu} u_{\varepsilon}\right\|_{L_{\mu}^{p}(X ; \mathbb{M})}^{p}<\infty,
$$

we have

$$
\varliminf_{\varepsilon \rightarrow 0} \int_{X} \Psi(x) L\left(x, \nabla_{\mu} u_{\varepsilon}\right) d \mu \geqslant \int_{X} \Psi(x) L\left(x, \nabla_{\mu} u\right) d \mu
$$

for all nonnegative measurable function $\Psi: X \longrightarrow[0, \infty]$.

We consider the following structure condition on $L: X \times \mathbb{M} \longrightarrow]-\infty, \infty]$ a Borel measurable integrand:

(Comp) there exist $s \in \mathbb{N}^{*}$, a finite family $\left\{f_{i}\right\}_{i=1}^{s}, f_{i}: X \times \mathbb{M} \longrightarrow[0, \infty]$ of $H_{\mu}^{1, p}$-quasiconvex Carathéodory integrands, and a convex and lower semicontinuous function $g$ : $\left.\left.X \times \mathbb{R}^{s} \longrightarrow\right]-\infty, \infty\right]$ such that for every $(x, \xi) \in X \times \mathbb{M}$

$$
\begin{aligned}
L(x, \xi) & =g\left(x, f_{1}(x, \xi), \cdots, f_{i}(x, \xi), \cdots, f_{s}(x, \xi)\right), \\
\partial g(x, t) & =\left\{\bar{\sigma} \in \mathbb{R}^{s}: \forall \tau \in \mathbb{R}^{s} \quad g(x, \tau) \geqslant g(x, t)+\langle\bar{\sigma}, \tau-t\rangle\right\} \subset \mathbb{R}_{+}^{s} \quad \text { for all } t \in \mathbb{R}^{s} .
\end{aligned}
$$

The condition on the subdifferential $\partial g(x, \cdot)$ of $g(x, \cdot)$ means that the convex function $g(x, \cdot)$ is nondecreasing with respect to each of its variables.

Theorem 8. Assume that $(X, d, \mu)$ satisfies $\left(\mathrm{AD}_{X}\right)$ and $\left(\mathrm{AL}_{X}\right)$. Let $\left.L: X \times \mathbb{M} \longrightarrow\right]-$ $\infty, \infty]$ be a Borel measurable function satisfying (Comp). Moreover, we assume that each 
$f_{i}$ satisfies q-polynomial growth with $\left.q \in\right] 1, p\left[\right.$, i.e. there exist $C_{i}>0$ and $A_{i} \in L_{\mu, \text { loc* }}^{1}(X)$ be such that for $\mu$-a.e. $x \in X$

$$
0 \leqslant f_{i}(x, \xi) \leqslant A_{i}(x)+C_{i}\left(1+|\xi|^{q}\right) \quad \text { for all } \xi \in \mathbb{M}
$$

Then for every $u,\left\{u_{\varepsilon}\right\}_{\varepsilon>0} \subset H_{\mu}^{1, p}\left(X ; \mathbb{R}^{m}\right)$ satisfying

$$
\lim _{\varepsilon \rightarrow 0}\left\|u_{\varepsilon}-u\right\|_{L_{\mu}^{p}\left(X ; \mathbb{R}^{m}\right)}=0 \quad \text { and } \quad \sup _{\varepsilon>0}\left\|\nabla_{\mu} u_{\varepsilon}\right\|_{L_{\mu}^{p}(X ; \mathbb{M})}^{p}<\infty,
$$

we have

$$
\varliminf_{\varepsilon \rightarrow 0} \int_{O} L\left(x, \nabla_{\mu} u_{\varepsilon}\right) d \mu \geqslant \int_{O} L\left(x, \nabla_{\mu} u\right) d \mu .
$$

for all open set $O \in \mathcal{O}(X)$.

Proof. Set $F(x, \xi):=\left(f_{1}(x, \xi), \cdots, f_{s}(x, \xi)\right) \in \mathbb{R}_{+}^{s}$ for all $(x, \xi) \in X \times \mathbb{M}$. Let $u \in$ $H_{\mu}^{1, p}\left(X ; \mathbb{R}^{m}\right)$ and $\left\{u_{n}\right\}_{n \in \mathbb{N}} \subset H_{\mu}^{1, p}\left(X ; \mathbb{R}^{m}\right)$ satisfying

$$
\lim _{n \rightarrow \infty}\left\|u_{n}-u\right\|_{L_{\mu}^{p}\left(X ; \mathbb{R}^{m}\right)}=0 \quad \text { and } \quad \sup _{n \in \mathbb{N}}\left\|\nabla_{\mu} u_{n}\right\|_{L_{\mu}^{p}(X ; \mathbb{M})}^{p}<\infty .
$$

By [Roc69, Corollary 4.6.], the multifunction $X \ni x \longmapsto \partial g\left(x, F\left(x, \nabla_{\mu} u(x)\right)\right)$ is measurable. By measurable selection theorem (see [CV77, Chap. III]) there exists a measurable function $\bar{\sigma}(\cdot)=\left(\bar{\sigma}_{1}(\cdot), \cdots, \bar{\sigma}_{s}(\cdot)\right)$ such that $\bar{\sigma}_{i} \geqslant 0$ for all $i \in\{1, \cdots, s\}$ and $\bar{\sigma}(x) \in \partial g\left(x, F\left(x, \nabla_{\mu} u(x)\right)\right)$ for all $x \in X$. It follows that for $\mu$-a.e. $x \in X$ and for every $n \in \mathbb{N}$ we have

$$
\begin{aligned}
L\left(x, \nabla_{\mu} u_{n}(x)\right) & =g\left(x, F\left(x, \nabla_{\mu} u_{n}(x)\right)\right) \\
& \geqslant g\left(x, F\left(x, \nabla_{\mu} u(x)\right)\right)+\left\langle\bar{\sigma}(x), F\left(x, \nabla_{\mu} u_{n}(x)\right)-F\left(x, \nabla_{\mu} u(x)\right)\right\rangle \\
& =L\left(x, \nabla_{\mu} u(x)\right)+\sum_{i=1}^{s} \bar{\sigma}_{i}(x)\left(f_{i}\left(x, \nabla_{\mu} u_{n}(x)\right)-f_{i}\left(x, \nabla_{\mu} u(x)\right)\right) .
\end{aligned}
$$

Apply Proposition 3 with $\Psi=\bar{\sigma}_{i}$, we have for every $i \in\{1, \cdots, s\}$

$$
\varliminf_{n \rightarrow \infty} \int_{O} \bar{\sigma}_{i}(x)\left(f_{i}\left(x, \nabla_{\mu} u_{n}\right)-f_{i}\left(x, \nabla_{\mu} u\right)\right) d \mu \geqslant 0 .
$$

Hence

$$
\begin{aligned}
\varliminf_{n \rightarrow \infty} \int_{O} L\left(x, \nabla_{\mu} u_{n}\right) d \mu & \geqslant \int_{O} L\left(x, \nabla_{\mu} u\right) d \mu+\sum_{i=1}^{s} \underline{\lim _{n \rightarrow \infty}} \int_{O} \bar{\sigma}_{i}(x)\left(f_{i}\left(x, \nabla_{\mu} u_{n}\right)-f_{i}\left(x, \nabla_{\mu} u\right)\right) d \mu \\
& \geqslant \int_{O} L\left(x, \nabla_{\mu} u\right) d \mu .
\end{aligned}
$$

As a consequence of Theorem 8 , we obtain an extension for $x$-dependent convex function $g$ of Proposition 3 .

Corollary 2. We assume that the integrand

$$
X \times \mathbb{M} \ni(x, \xi) \longmapsto L(x, \xi)=g\left(x, f_{1}(x, \xi), \cdots, f_{i}(x, \xi), \cdots, f_{s}(x, \xi)\right) \in[0, \infty]
$$

is p-coercive and that the hypotheses of Theorem 8 hold. Then $L$ is $H_{\mu}^{1, p}$-quasiconvex at every $\xi \in \mathbb{M}$ satisfying $L(\cdot, \xi) \in L_{\mu, \text { loc* }}^{1}(X)$. 
Proof of Lemma 3. Let $u \in H_{\mu}^{1, p}\left(X ; \mathbb{R}^{m}\right)$ and let $\left\{u_{\varepsilon}\right\}_{\varepsilon>0} \subset H_{\mu}^{1, p}\left(X ; \mathbb{R}^{m}\right)$ be a sequence such that $\lim _{\varepsilon \rightarrow 0}\left\|u_{\varepsilon}-u\right\|_{L_{\mu}^{p}\left(X ; \mathbb{R}^{m}\right)}=0$ and $\sup _{\varepsilon>0}\left\|\nabla_{\mu} u_{\varepsilon}\right\|_{L_{\mu}^{p}(X ; \mathbb{M})}^{p}<\infty$. Let $\Psi: X \longrightarrow[0, \infty]$ be a nonnegative measurable function.

Assume first that $\Psi=\mathbb{1}_{M}$ with $M \subset X$ a measurable set with finite measure. Let $\delta>0$. Since $\mu$ is Borel regular, there exists an open set $O_{\delta} \in \mathcal{O}(X)$ such that $O_{\delta} \supset M$ and $\mu\left(O_{\delta} \backslash M\right)+\int_{O_{\delta} \backslash M} A d \mu<\delta$. Since $\left.q \in\right] 1, p[$, for $\mu$-a.e. $x \in X$ we have $L(x, \xi) \leqslant$ $A(x)+2 C\left(1+|\xi|^{p}\right)$ for all $\xi \in \mathbb{M}$. Applying Theorem 5 we have

$$
\begin{aligned}
\varliminf_{\varepsilon \rightarrow 0} \int_{M} L\left(y, \nabla_{\mu} u_{\varepsilon}\right) d \mu & =\underline{\lim _{\varepsilon \rightarrow 0}}\left(\int_{O_{\delta}} L\left(y, \nabla_{\mu} u_{\varepsilon}\right) d \mu-\int_{O_{\delta} \backslash M} L\left(y, \nabla_{\mu} u_{\varepsilon}\right) d \mu\right) \\
& \geqslant \int_{O_{\delta}} L\left(y, \nabla_{\mu} u\right) d \mu-\varlimsup_{\varepsilon \rightarrow 0} \int_{O_{\delta} \backslash M} L\left(y, \nabla_{\mu} u_{\varepsilon}\right) d \mu \\
& \geqslant \int_{M} L\left(y, \nabla_{\mu} u\right) d \mu-\varlimsup_{\varepsilon \rightarrow 0} \int_{O_{\delta} \backslash M} L\left(y, \nabla_{\mu} u_{\varepsilon}\right) d \mu .
\end{aligned}
$$

We are reduced to show that $\overline{\lim }_{\delta \rightarrow 0} \varlimsup_{\lim _{\varepsilon \rightarrow 0}} I\left(u_{\varepsilon} ; O_{\delta} \backslash M\right)=0$. By (5.1) and Hölder inequality, we have for every $\varepsilon>0$ and $\delta>0$

$$
\begin{aligned}
\int_{O_{\delta} \backslash M} L\left(y, \nabla_{\mu} u_{\varepsilon}\right) d \mu & \leqslant \int_{O_{\delta} \backslash M} A d \mu+C \mu\left(O_{\delta} \backslash M\right)+\int_{O_{\delta} \backslash M}\left|\nabla_{\mu} u_{\varepsilon}\right|^{q} d \mu \\
& \leqslant \max (1, C) \delta+\left\|\nabla_{\mu} u_{\varepsilon}\right\|_{L_{\mu}^{p}(X ; \mathbb{M})}^{q} \mu\left(O_{\delta} \backslash M\right)^{1-\frac{q}{p}} \\
& \leqslant \max (1, C) \delta+\left(\sup _{\varepsilon>0}\left\|\nabla_{\mu} u_{\varepsilon}\right\|_{L_{\mu}^{p}(X ; \mathbb{M})}\right)^{q} \delta^{1-\frac{q}{p}} .
\end{aligned}
$$

Therefore $\lim _{\delta \rightarrow 0} \sup _{\varepsilon>0} I\left(u_{\varepsilon} ; O_{\delta} \backslash M\right)=0$. Now, if $M \subset X$ has not necessary finite measure, since $X$ can be written as a nondecreasing union of open sets with finite measure $X=\bigcup_{s \in \mathbb{N}} O_{s}$, we apply the previous argument to $M \cap O_{s}$ which has finite measure and is contained in $M$. Letting $s \rightarrow \infty$ and using the monotone convergence theorem we obtain

$$
\varliminf_{\varepsilon \rightarrow 0} \int_{X} \mathbb{1}_{M}(y) L\left(y, \nabla_{\mu} u_{\varepsilon}\right) d \mu \geqslant \int_{X} \mathbb{1}_{M}(y) L\left(y, \nabla_{\mu} u\right) d \mu .
$$

Now, assume that $\Psi$ is a nonnegative simple function, i.e. $\Psi:=\sum_{i \in I} a_{i} \mathbb{1}_{M_{i}}$ for some finite family of nonnegative real numbers $\left\{a_{i}\right\}_{i \in I} \subset \mathbb{R}_{+}$and some finite family of mutually disjoints measurable sets $\left\{M_{i}\right\}_{i \in I}$ of $X$. Using (5.3) we have

$$
\begin{aligned}
\underline{\lim _{\varepsilon \rightarrow 0}} \int_{X} \Psi(x) L\left(x, \nabla_{\mu} u_{\varepsilon}\right) d \mu & =\varliminf_{\varepsilon \rightarrow 0} \sum_{i \in I} \int_{M_{i}} a_{i} L\left(x, \nabla_{\mu} u_{\varepsilon}\right) d \mu \\
& \geqslant \sum_{i \in I} a_{i} \varliminf_{\varepsilon \rightarrow 0} \int_{M_{i}} L\left(x, \nabla_{\mu} u_{\varepsilon}\right) d \mu \\
& \geqslant \sum_{i \in I} a_{i} \int_{M_{i}} L\left(x, \nabla_{\mu} u\right) d \mu=\int_{X} \Psi(x) L\left(x, \nabla_{\mu} u\right) d \mu .
\end{aligned}
$$

If $\Psi$ is a nonnegative bounded measurable function, there exists a sequence of nonnegative simple functions $\left\{\Psi_{k}\right\}_{k \in \mathbb{N}}$ such that $\lim _{k \rightarrow \infty}\left\|\Psi_{k}-\Psi\right\|_{L_{\mu}^{\infty}(X)}=0$. Consider a nondecreasing union of open sets with finite measure such that $X=\bigcup_{s \in \mathbb{N}} O_{s}$. Since the growth condition (5.1) we have for every $s \in \mathbb{N}$

$$
\varlimsup_{k \rightarrow \infty} \sup _{\varepsilon>0} \int_{O_{s}}\left|\Psi(x)-\Psi_{k}(x)\right| L\left(x, \nabla_{\mu} u_{\varepsilon}\right) d \mu=0 .
$$


Now, for every $k, s \in \mathbb{N}$ we have

$$
\begin{aligned}
& \underline{\lim _{\varepsilon \rightarrow 0}} \int_{X} \Psi(x) L\left(x, \nabla_{\mu} u_{\varepsilon}\right) d \mu \\
& \geqslant-\sup _{\varepsilon>0} \int_{O_{s}}\left|\Psi(x)-\Psi_{k}(x)\right| L\left(x, \nabla_{\mu} u_{\varepsilon}\right) d \mu+\int_{O_{s}} \Psi_{k}(x) L\left(x, \nabla_{\mu} u\right) d \mu .
\end{aligned}
$$

Letting $k \rightarrow \infty$ and using Fatou Lemma we obtain

$$
\varliminf_{\varepsilon \rightarrow 0} \int_{X} \Psi(x) L\left(x, \nabla_{\mu} u_{\varepsilon}\right) d \mu \geqslant \varliminf_{k \rightarrow \infty} \int_{O_{s}} \Psi_{k}(x) L\left(x, \nabla_{\mu} u\right) d \mu \geqslant \int_{O_{s}} \Psi(x) L\left(x, \nabla_{\mu} u\right) d \mu,
$$

letting $s \rightarrow \infty$ we have by the monotone convergence theorem

$$
\varliminf_{\varepsilon \rightarrow 0} \int_{X} \Psi(x) L\left(x, \nabla_{\mu} u_{\varepsilon}\right) d \mu \geqslant \int_{X} \Psi(x) L\left(x, \nabla_{\mu} u\right) d \mu .
$$

In case $\Psi$ not bounded, set $\Psi_{n}:=\min (\Psi, n)$ for all $n \in \mathbb{N}$. Using (5.4) we have for every $n \in \mathbb{N}$

$$
\varliminf_{\varepsilon \rightarrow 0} \int_{X} \Psi(x) L\left(x, \nabla_{\mu} u_{\varepsilon}\right) d \mu \geqslant \varliminf_{\varepsilon \rightarrow 0} \int_{X} \Psi_{n}(x) L\left(x, \nabla_{\mu} u_{\varepsilon}\right) d \mu \geqslant \int_{X} \Psi_{n}(x) L\left(x, \nabla_{\mu} u\right) d \mu,
$$

letting $n \rightarrow \infty$ and using Fatou lemma we obtain the desired result.

Remark 3 (Quasiaffine integrands). It can be interesting to extend, in the setting of metric measure spaces, some notions of the calculus of variations. For instance, the concept of quasiaffine integrands can be stated as follows: we say that a real valued Borel measurable integrand $L: X \times \mathbb{M} \longrightarrow \mathbb{R}$ is $H_{\mu}^{1, p}$-quasiaffine at $\xi \in \mathbb{M}$ if $L$ and $-L$ are $H_{\mu}^{1, p}$-quasiconvex at $\xi$, i.e if for $\mu$-a.e. $x \in X$ we have

$$
\begin{aligned}
& L(x, \xi) \leqslant \lim _{\rho \rightarrow 0} \inf _{\varphi \in H_{\mu, 0}^{1, p}\left(B_{\rho}(x) ; \mathbb{R}^{m}\right)} f_{\bar{B}_{\rho}(x)} L\left(y, \xi+\nabla_{\mu} \varphi\right) d \mu \\
& L(x, \xi) \geqslant \varlimsup_{\rho \rightarrow 0} \sup _{\varphi \in H_{\mu, 0}^{1, p}\left(B_{\rho}(x) ; \mathbb{R}^{m}\right)} f_{\bar{B}_{\rho}(x)} L\left(y, \xi+\nabla_{\mu} \varphi\right) d \mu .
\end{aligned}
$$

That means

$$
\begin{aligned}
L(x, \xi) & =\lim _{\rho \rightarrow 0} \inf _{\varphi \in H_{\mu, 0}^{1, p}\left(B_{\rho}(x) ; \mathbb{R}^{m}\right)} f_{\bar{B}_{\rho}(x)} L\left(y, \xi+\nabla_{\mu} \varphi\right) d \mu \\
& =\lim _{\rho \rightarrow 0} \sup _{\varphi \in H_{\mu, 0}^{1, p}\left(B_{\rho}(x) ; \mathbb{R}^{m}\right)} f_{\bar{B}_{\rho}(x)} L\left(y, \xi+\nabla_{\mu} \varphi\right) d \mu
\end{aligned}
$$

We say that $L$ is $H_{\mu}^{1, p}$-quasiaffine if it is $H_{\mu}^{1, p}$-quasiaffine at every $\xi \in \mathbb{M}$.

It is well known that, in the Euclidean setting, a necessary condition for the weak continuity of the integral associated with $L$ (not depending on $x$ ) is that $L$ is quasiaffine, i.e. $L$ and $-L$ are quasiconvex, see for instance [Dac08, Theorem 8.19, pp. 393]. We do not know how to prove a similar result in the setting of metric measure spaces. However, for the sufficiency of the condition we have:

Proposition 4. Assume that $\mu$ is finite and $L$ is a Carathéodory integrand which is $H_{\mu}^{1, p}$ quasiaffine. Assume that $|L|$ has q-polynomial growth with $q \in] 1, p[$, i.e. for some $C>0$ it holds $|L(x, \xi)| \leqslant C\left(1+|\xi|^{q}\right)$ for all $(x, \xi) \in X \times \mathbb{M}$. Then for every $u \in H_{\mu}^{1, p}\left(X ; \mathbb{R}^{m}\right)$ and 
every sequence $\left\{u_{\varepsilon}\right\}_{\varepsilon>0} \subset H_{\mu}^{1, p}\left(X ; \mathbb{R}^{m}\right)$ satisfying $\sup _{\varepsilon>0}\left\|\nabla_{\mu} u_{\varepsilon}\right\|_{L_{\mu}^{p}}<\infty$ and $\lim _{\varepsilon \rightarrow 0} \| u_{\varepsilon}-$ $u \|_{L_{\mu}^{p}}=0$ we have

$$
\lim _{\varepsilon \rightarrow 0} \int_{O} L\left(x, \nabla_{\mu} u_{\varepsilon}\right) d \mu=\int_{O} L\left(x, \nabla_{\mu} u\right) d \mu \quad \text { for all } O \in \mathcal{O}(X) .
$$

Proof. Let $u \in H_{\mu}^{1, p}\left(X ; \mathbb{R}^{m}\right)$ and $\left\{u_{\varepsilon}\right\}_{\varepsilon>0} \subset H_{\mu}^{1, p}\left(X ; \mathbb{R}^{m}\right)$ be such that $\sup _{\varepsilon>0}\left\|\nabla_{\mu} u_{\varepsilon}\right\|_{L_{\mu}^{p}}<$ $\infty$ and $\lim _{\varepsilon \rightarrow 0}\left\|u_{\varepsilon}-u\right\|_{L_{\mu}^{p}}=0$. Following one approximation argument of [BZ90, pp. 371], we set $L_{k}(x, \xi):=k+\max (L(x, \xi),-k)$ for all $k \in \mathbb{N}, x \in X$ and $\xi \in \mathbb{M}$. By Remarks 1 (a) the Carathéodory integrands $L_{k}$ are $H_{\mu}^{1, p}$-quasiconvex and it is easy to check that $0 \leqslant L_{k}(x, \xi) \leqslant k+2 C\left(1+|\xi|^{p}\right)$ for all $k \in \mathbb{N}, x \in X$ and $\xi \in \mathbb{M}$. Apply Theorem 5 to each $L_{k}$ we get for every $k \geqslant C+1$

$$
\begin{aligned}
\frac{\lim }{\varepsilon \rightarrow 0} \int_{O} L\left(x, \nabla_{\mu} u_{\varepsilon}\right) d \mu & \geqslant \frac{\lim }{\varepsilon \rightarrow 0} \int_{O} L_{k}\left(x, \nabla_{\mu} u_{\varepsilon}\right) d \mu+\underline{\lim } \int_{\varepsilon \rightarrow 0}\left(L-L_{k}\right)\left(x, \nabla_{\mu} u_{\varepsilon}\right) d \mu \\
& \geqslant \int_{O} L_{k}\left(x, \nabla_{\mu} u\right) d \mu+\underline{\lim } \int_{O \cap\left[L\left(\cdot, \nabla_{\mu} u_{\varepsilon}(\cdot)\right)+k \leqslant 0\right]} L\left(x, \nabla_{\mu} u_{\varepsilon}(x)\right)+k d \mu .
\end{aligned}
$$

Noticing that $L_{k} \geqslant L$ and using the $q$-growth we have

$$
\varliminf_{\varepsilon \rightarrow 0} \int_{O} L\left(x, \nabla_{\mu} u_{\varepsilon}\right) d \mu \geqslant \int_{O} L\left(x, \nabla_{\mu} u\right) d \mu-\Delta_{u}
$$

where

$$
\Delta_{u}:=\varlimsup_{k \rightarrow \infty} \sup _{\varepsilon>0} \int_{\left[\left|\nabla_{\mu} u_{\varepsilon}\right| q \geqslant \frac{k-C}{C}\right]} k+C\left(1+\left|\nabla_{\mu} u_{\varepsilon}\right|^{q}\right) d \mu .
$$

We have $\Delta_{u}=0$ since $q<p$ and $\sup _{\varepsilon>0}\left\|\nabla_{\mu} u_{\varepsilon}\right\|_{L_{\mu}^{p}}<\infty$. Now, apply the previous arguments with $-L$ in place of $L$ we get $(5.6)$.

\section{On the Finiteness CONDition $L(\cdot, \xi) \in L_{\mu}^{1}(X)$}

When for instance $X$ is compact, in Theorem 2 and Theorem 3 , the finiteness condition $L(\cdot, \xi) \in L_{\mu}^{1}(X)$ is needed to conclude that $H_{\mu}^{1, p}$-quasiconvexity of $L$ at $\xi$ is necessary for the lower semicontinuity. Proposition 5 below gives an indication when $L(\cdot, \xi) \in L_{\mu}^{1}(X)$ is not satisfied.

For each $\xi \in \mathbb{M}$ and $l \in \mathbb{N} \cup\{\infty\}$, we set, for a Borel measurable integrand $L$ : $X \times \mathbb{M} \longrightarrow[0, \infty]$,

$$
V_{\xi}^{l}:=[\bar{L}(\cdot, \xi)<l] \text { where } \bar{L}(x, \xi):=\underline{\lim }_{\rho \rightarrow 0} \inf _{\varphi \in H_{\mu, 0}^{1, p}\left(B_{\rho}(x) ; \mathbb{R}^{m}\right)} f_{\bar{B}_{\rho}(x)} L\left(y, \xi+\nabla_{\mu} \varphi\right) d \mu .
$$

It is easy to see that the family $\left\{V_{\xi}^{l}\right\}_{l \in \mathbb{N}}$ is nondecreasing and $\bigcup_{l \in \mathbb{N}} V_{\xi}^{l}=V_{\xi}^{\infty}$.

Proposition 5. Assume that $\mu$ is finite and $X$ is proper. Let $L: X \times \mathbb{M} \longrightarrow[0, \infty]$ be a p-coercive Borel measurable integrand. Assume that for every $u,\left\{u_{\varepsilon}\right\}_{\varepsilon>0} \subset H_{\mu}^{1, p}\left(X ; \mathbb{R}^{m}\right)$ satisfying $\lim _{\varepsilon \rightarrow 0}\left\|u_{\varepsilon}-u\right\|_{L_{\mu}^{p}\left(X ; \mathbb{R}^{m}\right)}=0$, it holds

$$
\varliminf_{\varepsilon \rightarrow 0} \int_{O} L\left(x, \nabla_{\mu} u_{\varepsilon}\right) d \mu \geqslant \int_{O} L\left(x, \nabla_{\mu} u\right) d \mu \quad \text { for all open set } O \in \mathcal{O}(X) .
$$

Let $\xi \in \mathbb{M}$ be such that $\bar{L}(\cdot, \xi)$ is upper semicontinuous. Then $L(\cdot, \xi) \in L_{\mu, \text { loc }}^{1}\left(V_{\xi}^{\infty}\right)$ whenever $\mu\left(V_{\xi}^{\infty}\right)>0$. 
Proof. Let $\xi \in \mathbb{M}$ be such that $\bar{L}(\cdot, \xi)$ is upper semicontinuous. Assume that $\mu\left(V_{\xi}^{\infty}\right)>0$. There is $l_{0} \in \mathbb{N}$ such that $\mu\left(V_{\xi}^{l}\right)>0$ for all $l \geqslant l_{0}$, note that $V_{\xi}^{l}$ is open since $\bar{L}(\cdot, \xi)$ is upper semicontinuous. Assume for the moment that we have proved that $\int_{V_{\xi}^{l}} L(y, \xi) d \mu \leqslant$ $l$ for all $l \geqslant l_{0}$, then since every compact subset $T \subset V_{\xi}^{\infty}$ satisfies $T \subset V_{\xi}^{l}$ for some $l \geqslant l_{0}$, we have $L(\cdot, \xi) \in L_{\mu}^{1}(T)$.

Let $l \geqslant l_{0}$. Let us show that $\int_{V_{\xi}^{l}} L(y, \xi) d \mu \leqslant l$. Fix $(n, k) \in \mathbb{N}^{*} \times \mathbb{N}^{*}$. For each $x \in V_{\xi}^{l}$ there exist $\left.\rho_{k, n}^{x} \in\right] 0,\left(k 2^{n}\right)^{-\frac{1}{p}}\left[\right.$ and $\varphi_{k, n}^{x} \in H_{\mu, 0}^{1, p}\left(B_{\rho_{k, n}^{x}}(x) ; \mathbb{R}^{m}\right)$ such that $\bar{B}_{\rho_{k, n}^{x}}(x) \subset V_{\xi}^{l}$ and

$$
\frac{1}{k \mu(X)}+l \geqslant f_{\bar{B}_{\rho_{k, n}^{x}}^{x}(x)} L\left(y, \xi+\nabla_{\mu} \varphi_{k, n}^{x}\right) d \mu \geqslant c f_{\bar{B}_{\rho_{k, n}^{x}}^{x}(x)}\left|\xi+\nabla_{\mu} \varphi_{k, n}^{x}\right|^{p} d \mu
$$

where we have used the $p$-coercivity assumption. The family $\left\{\bar{B}_{\rho_{k, n}^{x}}(x)\right\}_{x \in V_{\xi}^{l}, n \in \mathbb{N}^{*}}$ of closed balls is a fine cover of $V_{\xi}^{l}$, so by Vitali covering theorem there exists a countable family of mutually disjoint closed balls $\left\{\bar{B}_{\rho_{k, n_{i}}^{x_{i}}}\left(x_{i}\right)\right\}_{i \in \mathbb{N}}$ such that $\mu\left(V_{\xi}^{l} \backslash \bigcup_{i=0}^{\infty} \bar{B}_{\rho_{k, n_{i}}^{x_{i}}}\left(x_{i}\right)\right)=0$. We set $\varphi_{k}^{i}:=\varphi_{k, n_{i}}^{x_{i}}, B_{k}^{i}:=\bar{B}_{\rho_{k, n_{i}}^{x_{i}}}\left(x_{i}\right), \rho_{k}^{i}:=\rho_{k, n_{i}}^{x_{i}}$ for all $i \in \mathbb{N}$, and $\varphi_{k}:=\sum_{i \in \mathbb{N}} \varphi_{k}^{i} \mathbb{1}_{B_{k}^{i}} \in$ $H_{\mu, 0}^{1, p}\left(X ; \mathbb{R}^{m}\right)$. Assume for the moment that the sequence $\left\{\varphi_{k}\right\}_{k \in \mathbb{N}^{*}} \subset H_{\mu, 0}^{1, p}\left(X ; \mathbb{R}^{m}\right)$ is converging to 0 in $L_{\mu}^{p}\left(X ; \mathbb{R}^{m}\right)$, i.e. $\lim _{k \rightarrow \infty}\left\|\varphi_{k}\right\|_{L_{\mu}^{p}}=0$. Set $F_{k}:=\bigcup_{i \in \mathbb{N}} B_{k}^{i}$ and $F_{\infty}:=$ $\bigcap_{k \in \mathbb{N}^{*}} F_{k} \in \mathcal{B}(X)$. We see that $\mu\left(V_{\xi}^{l}\right)=\mu\left(F_{\infty} \cap V_{\xi}^{l}\right)$. Take the sum over all closed balls $B_{k}^{i}$ in 6.2 , we obtain

$$
\frac{1}{k}+l \geqslant \int_{F_{k}} L\left(y, \xi+\nabla_{\mu} \varphi_{k}\right) d \mu \geqslant \int_{F_{\infty}} L\left(y, \xi+\nabla_{\mu} \varphi_{k}\right) d \mu=\int_{V_{\xi}^{l}} L\left(y, \xi+\nabla_{\mu} \varphi_{k}\right) d \mu .
$$

letting $k \rightarrow \infty$ and using 6.1 we find that $\int_{V_{\xi}^{l}} L(y, \xi) d \mu \leqslant l$.

It remains to show that $\lim _{k \rightarrow \infty}\left\|\varphi_{k}\right\|_{L_{\mu}^{p}}=0$. By using the Sobolev inequality 2.5) and the coercivity condition, we have

$$
\begin{aligned}
\int_{X}\left|\varphi_{k}\right|^{p} d \mu & \leqslant \sum_{i=0}^{\infty}\left(\rho_{k}^{i}\right)^{p} C_{S}^{p} \int_{B_{k}^{i}}\left|\nabla_{\mu} \varphi_{k}^{i}\right|^{p} d \mu \\
& \leqslant \sum_{i=0}^{\infty}\left(\rho_{k}^{i}\right)^{p} 2^{p-1} C_{S}^{p}\left(\int_{B_{k}^{i}}\left|\xi+\nabla_{\mu} \varphi_{k}^{i}\right|^{p} d \mu+\int_{B_{k}^{i}}|\xi|^{p} d \mu\right) \\
& \leqslant \sum_{i=0}^{\infty}\left(\rho_{k}^{i}\right)^{p} 2^{p-1} C_{S}^{p}\left(\int_{B_{k}^{i}} \frac{1}{c} L\left(y, \xi+\nabla_{\mu} \varphi_{k}^{i}\right) d \mu+\int_{B_{k}^{i}}|\xi|^{p} d \mu\right) \\
& \leqslant 2^{p-1} \frac{C_{S}^{p}}{c} \sum_{i=0}^{\infty}\left(\rho_{k}^{i}\right)^{p}\left(\int_{B_{k}^{i}} l+\frac{1}{k \mu(X)} d \mu+c|\xi|^{p} \mu\left(B_{k}^{i}\right)\right) \\
& \leqslant 2^{p-1} \frac{C_{S}^{p}}{c}\left(l \mu(X)+1+c|\xi|^{p} \mu(X)\right) \frac{2}{k} .
\end{aligned}
$$

By passing to the limit $k \rightarrow \infty$, we obtain that $\varphi_{k} \longrightarrow 0$ in $L_{\mu}^{p}\left(X ; \mathbb{R}^{m}\right)$.

As an illustration of Proposition 5 we have:

Corollary 3. Assume that $X$ is compact. Let $L: X \times \mathbb{M} \longrightarrow[0, \infty]$ be a p-coercive Borel measurable integrand. Assume that for every $u,\left\{u_{\varepsilon}\right\}_{\varepsilon>0} \subset H_{\mu}^{1, p}\left(X ; \mathbb{R}^{m}\right)$ satisfying 
$\lim _{\varepsilon \rightarrow 0}\left\|u_{\varepsilon}-u\right\|_{L_{\mu}^{p}\left(X ; \mathbb{R}^{m}\right)}=0$, it holds

$$
\varliminf_{\varepsilon \rightarrow 0} \int_{O} L\left(x, \nabla_{\mu} u_{\varepsilon}\right) d \mu \geqslant \int_{O} L\left(x, \nabla_{\mu} u\right) d \mu
$$

for all open set $O \in \mathcal{O}(X)$.

Assume that $\bar{L}(\cdot, \xi)$ is continuous for all $\xi \in \mathbb{M}$, and that there exists $L_{0}: \mathbb{M} \longrightarrow[0, \infty]$ a Borel measurable function such that for some $\alpha, \beta>0$ we have for $\mu$-a.e. $x \in X$

$$
\alpha L_{0}(\xi) \leqslant \bar{L}(x, \xi) \leqslant \beta\left(1+L_{0}(\xi)\right) \quad \text { for all } \xi \in \mathbb{M} \text {. }
$$

Then $L$ is $H_{\mu}^{1, p}$-quasiconvex.

Proof. Let $\xi \in \mathbb{M}$. If $L_{0}(\xi)<\infty$ then $\mu\left(X \backslash V_{\xi}^{\infty}\right)=0$. By Proposition 5 we have $L(\cdot, \xi) \in L_{\mu, \mathrm{loc}}^{1}\left(V_{\xi}^{\infty}\right)=L_{\mu, \mathrm{loc}}^{1}(X)=L_{\mu}^{1}(X)$ since $X$ is compact, and so Theorem 3 gives that $L$ is $H_{\mu}^{1, p}$-quasiconvex at $\xi$. Otherwise, $L_{0}(\xi)=\infty$ hence $\infty=\bar{L}(x, \xi) \geqslant L(x, \xi)$ for $\mu$-a.a. $x \in X$.

\section{Proofs of Proposition 1 and Theorem 4}

Proof of Proposition 1 .

Proof of $(i)$. Since $\mu$ is doubling, $X$ satisfies the Vitali covering theorem see [HKST15, Theorem 3.4.3, pp. 73].

Proof of (ii). The closability of the $\mu$-gradient in $\operatorname{Lip}\left(X ; \mathbb{R}^{m}\right)$, given by Theorem 1 (iv), can be extended from $\operatorname{Lip}\left(X ; \mathbb{R}^{m}\right)$ to $H_{\mu}^{1, p}\left(X ; \mathbb{R}^{m}\right)$ by using the closability theorem of Franchi, Hajłasz and Koskela (see [FHK99, Theorem 10]).

Proof of (iii). According to [BB11, Corollary 4.24 pp. 93], since $\mu$ is doubling and $X$ supports a $(1, p)$-Poincaré inequality, we can assert that $X$ supports a $(p, p)$-Poincaré inequality, i.e. there exist $C_{p}>0$ and $\sigma \geqslant 1$ such that for every $x \in X$ and every $\rho>0$,

$$
\left(f_{B_{\rho}(x)}\left|f(y)-f_{B_{\rho}(x)} f d \mu\right|^{p} d \mu(y)\right)^{\frac{1}{p}} \leqslant \rho C_{p}\left(f_{B_{\sigma \rho}(x)} g^{p} d \mu\right)^{\frac{1}{p}}
$$

for every $f \in L_{\mu}^{p}(X)$ and every $p$-weak upper gradient $g \in L_{\mu}^{p}(X)$ for $f$. Now, we can use the Sobolev inequality in [BB11, Theorem 5.51, pp. 142] to assert that there exists $C_{S}>0$ such that

$$
\left(f_{B_{\rho}(x)}|w|^{p} d \mu\right)^{\frac{1}{p}} \leqslant \rho C_{S}\left(f_{B_{\rho}(x)} g_{w}^{p} d \mu\right)^{\frac{1}{p}}
$$

for all $0<\rho \leqslant \rho_{0}$, with $\rho_{0}>0$, all $w \in H_{\mu, 0}^{1, p}\left(B_{\rho}(x)\right)$, and where $g_{w}$ is the minimal $p$-weak upper gradient for $w$. Moreover (see [Che99, §4] and also [BB11, §B.2, pp. 363], Bjö00 and [GH13, Remark 2.15]), there exists $\alpha \geqslant 1$ such that for every $w \in H_{\mu}^{1, p}(X)$ and $\mu$-a.e. $x \in X$,

$$
\frac{1}{\alpha}\left|g_{w}(x)\right| \leqslant\left|D_{\mu} w(x)\right| \leqslant \alpha\left|g_{w}(x)\right|,
$$

where $D_{\mu}$ corresponds to $\nabla_{\mu}$ with $m=1$. As for $v=\left(v_{i}\right)_{i=1, \cdots, m} \in H_{\mu}^{1, p}\left(X ; \mathbb{R}^{m}\right)$ we have $\nabla_{\mu} v=\left(D_{\mu} v_{i}\right)_{i=1, \cdots, m}$, it follows that

$$
\frac{1}{\alpha}\left|g_{v}(x)\right| \leqslant\left|\nabla_{\mu} v(x)\right| \leqslant \alpha\left|g_{v}(x)\right|
$$

for $\mu$-a.a. $x \in X$, where $g_{v}:=\left(g_{v_{i}}\right)_{i=1, \cdots, m}$ is naturally called the minimal $p$-weak upper gradient for $v$. Combining 7.2 with 7.3 we obtain 2.5. 
Proof of $[$ iv). From Björn (see [Bjö00, Theorem 4.5 and Corollary 4.6] and also [GH13, Theorem 2.12]) we see that for every $k \in \mathbb{N}$, every $u \in H_{\mu}^{1, p}\left(X ; \mathbb{R}^{m}\right)$ and $\mu$-a.e. $x \in X_{k}$,

$$
\nabla_{\mu} u_{x}(y)=\nabla_{\mu} u(x) \text { for } \mu \text {-a.a. } y \in X_{k},
$$

where $u_{x} \in H_{\mu}^{1, p}\left(X ; \mathbb{R}^{m}\right)$ is given by

$$
u_{x}(y):=u(x)+\nabla_{\mu} u(x) \cdot\left(\gamma^{k}(y)-\gamma^{k}(x)\right)
$$

and $u$ is $L_{\mu}^{p}$-differentiable at $x$, i.e.,

$$
\lim _{\rho \rightarrow 0} \frac{1}{\rho}\left\|u-u_{x}\right\|_{L_{\mu}^{p}\left(B_{\rho}(x) ; \mathbb{R}^{m}\right)}=0 .
$$

Proof of $[(v)$. For every $\rho>0, t \in] 0,1[$ and every $x \in X$, there exists a function $\varphi \in$ $\operatorname{Lip}(X ;[0,1])$ such that

$$
\varphi(x)=0 \text { for all } x \in X \backslash B_{\rho}(x), \varphi(x)=1 \text { for all } x \in \bar{B}_{\tau \rho}(x)
$$

and $\|\operatorname{Lip} \varphi\|_{L_{\mu}^{\infty}(X)} \leqslant \frac{1}{\rho(1-t)}$ where $\operatorname{Lip} \varphi(y):=\varlimsup_{d(y, z) \rightarrow 0} \frac{|\varphi(y)-\varphi(z)|}{d(y, z)}$ for all $y \in X$.

But, since $\mu$ is doubling and $X$ supports a $(1, p)$-Poincaré inequality, from Cheeger (see Che99, Theorem 6.1]) we have $\operatorname{Lip} \varphi(y)=g_{\varphi}(y)$ for $\mu$-a.a. $y \in X$, where $g_{\varphi}$ is the minimal $p$-weak upper gradient for $\varphi$. Hence

$$
\left\|D_{\mu} \varphi\right\|_{L_{\mu}^{\infty}\left(X ; \mathbb{R}^{N}\right)} \leqslant \frac{\alpha}{\rho(1-t)}
$$

because $\left|D_{\mu} \varphi(y)\right| \leqslant \alpha\left|g_{\varphi}(y)\right|$ for $\mu$-a.a. $y \in X$.

Proof of Theorem 4. Let $u \in H_{\mu}^{1, p}\left(X ; \mathbb{R}^{m}\right)$ be such that $(3.12)$ holds. By $\left(\mathrm{C}_{1}\right)$ and (3.12), the theorem of differentiation of measures (see [HKST15, pp. 82]) gives for $\mu$-a.e. $x \in X$

$$
\frac{d I(u ; \cdot)}{d \mu}(x)=\lim _{\rho \rightarrow 0} \frac{I\left(u ; \bar{B}_{\rho}(x)\right)}{\mu\left(\bar{B}_{\rho}(x)\right)}<\infty .
$$

Let $\alpha>0$. We have to show that $\mu\left(N_{\alpha}\right)=0$ where

$$
N_{\alpha}:=\left\{x \in X: \lim _{\rho \rightarrow 0} \inf _{\varphi \in H_{\mu, 0}^{1, p}\left(B_{\rho}(x) ; \mathbb{R}^{m}\right)} \frac{I\left(u+\varphi ; \bar{B}_{\rho}(x)\right)}{\mu\left(\bar{B}_{\rho}(x)\right)}-\frac{I\left(u ; \bar{B}_{\rho}(x)\right)}{\mu\left(\bar{B}_{\rho}(x)\right)}<-\alpha\right\} .
$$

Since $X$ is a countable union of balls with finite measure, we can write $N_{\alpha}=\bigcup_{s=0}^{\infty} B_{s} \cap N_{\alpha}$ where $B_{s}$ is an open ball and $\mu\left(B_{s}\right)<\infty$, so it is enough to prove that $\mu\left(N_{\alpha}^{s}\right)=0$ with $N_{\alpha}^{s}:=B_{s} \cap N_{\alpha}$ for all $s \in \mathbb{N}$.

Fix $s \in \mathbb{N}$. For each $x \in N_{\alpha}^{s}$ there exists $\left.\rho^{x} \in\right] 0,1\left[\operatorname{such}\right.$ that $\bar{B}_{\rho^{x}}(x) \subset B_{s}$. Fix $(k, n) \in \mathbb{N}^{*} \times \mathbb{N}^{*}$. For each $x \in N_{\alpha}^{s}$ there exist $\left.\rho_{k, n}^{x} \in\right] 0, \min \left(\left(\frac{1}{k 2^{n}}\right)^{\frac{1}{p}}, \rho^{x}\right)\left[\right.$ and $\varphi_{k, n}^{x} \in$ $H_{\mu, 0}^{1, p}\left(B_{\rho_{k, n}^{x}}(x) ; \mathbb{R}^{m}\right)$ such that

$$
\frac{1}{\mu\left(\bar{B}_{\rho_{k, n}^{x}}(x)\right)}\left[I\left(u+\varphi_{k, n}^{x} ; \bar{B}_{\rho_{k, n}^{x}}(x)\right)-I\left(u ; \bar{B}_{\rho_{k, n}^{x}}(x)\right)\right]<-\alpha+\frac{1}{k \mu\left(B_{s}\right)} .
$$

The family of closed balls $\left\{\bar{B}_{\rho_{k, n}^{x}}(x)\right\}_{x \in N_{\alpha}^{s}, n \in \mathbb{N}^{*}}$ is a fine cover of $N_{\alpha}^{s}$, so by Vitali covering theorem there exists a countable family of mutually disjoint closed balls $\left\{\bar{B}_{\rho_{k, n_{i}}^{x_{i}}}\left(x_{i}\right)\right\}_{i \in \mathbb{N}}$ 
such that

$$
\mu\left(N_{\alpha}^{s} \backslash \bigcup_{i=0}^{\infty} \bar{B}_{\rho_{k, n_{i}}^{x_{i}}}\left(x_{i}\right)\right)=0 \quad \text { and } \bigcup_{i=0}^{\infty} \bar{B}_{\rho_{k, n_{i}}^{x_{i}}}\left(x_{i}\right) \subset B_{s} .
$$

We set $\varphi_{k}^{i}:=\varphi_{k, n_{i}}^{x_{i}}, B_{k}^{i}:=\bar{B}_{\rho_{k, n_{i}}^{x_{i}}}\left(x_{i}\right), \rho_{k}^{i}:=\rho_{k, n_{i}}^{x_{i}}$ for all $i \in \mathbb{N}$. Moreover, for each $l \in \mathbb{N}$ we set $\varphi_{k, l}:=\sum_{i=0}^{l} \varphi_{k}^{i} \mathbb{1}_{B_{k}^{i}} \in H_{\mu, 0}^{1, p}\left(B_{s} ; \mathbb{R}^{m}\right)$.

Fix $l \in \mathbb{N}$. We have by using the Sobolev inequality (2.5) and the coercivity condition $\left(\mathrm{C}_{2}\right)$

$$
\begin{aligned}
\int_{X}\left|\varphi_{k, l}(y)\right|^{p} d \mu \leqslant \sum_{i=0}^{\infty} \int_{B_{k}^{i}}\left|\varphi_{k}^{i}(y)\right|^{p} d \mu & \leqslant \sum_{i=0}^{\infty}\left(\rho_{k}^{i}\right)^{p} C_{S}^{p} \int_{B_{k}^{i}}\left|\nabla_{\mu} \varphi_{k}^{i}(y)\right|^{p} d \mu \\
& \leqslant \frac{2^{p-1} C_{S}^{p}}{c} \sum_{i=0}^{\infty}\left(\rho_{k}^{i}\right)^{p} I\left(u+\varphi_{k}^{i} ; B_{k}^{i}\right)+I\left(u ; B_{k}^{i}\right) \\
& \leqslant\left(\frac{2^{p-1} C_{S}^{p}}{c} \int_{B_{s}}-\alpha+\frac{1}{k \mu\left(B_{s}\right)} d \mu+2 I\left(u ; B_{s}\right)\right) \frac{2}{k} .
\end{aligned}
$$

Since $I\left(u ; B_{s}\right)<\infty$, by passing to the limit $k \rightarrow \infty$ we find that $\varphi_{k, l} \rightarrow 0$ in $L_{\mu}^{p}\left(X ; \mathbb{R}^{m}\right)$.

We set $F_{k}^{l}:=\bigcup_{i=0}^{l} B_{k}^{i} \subset O$ for all $l \in \mathbb{N}$. Let $\varepsilon>0$. Because of 7.5 there exists $l_{\varepsilon} \in \mathbb{N}$ such that $\mu\left(N_{\alpha}^{s} \backslash F_{k}^{l_{\varepsilon}}\right)<\frac{\varepsilon}{\alpha}$. Take the sum over the finite family of mutually closed balls $\left\{B_{k}^{i}\right\}_{i=0}^{l_{\varepsilon}}$ in (7.4), we obtain

$$
\sum_{i=0}^{l_{\varepsilon}} I\left(u+\varphi_{k}^{i} ; B_{k}^{i}\right)-I\left(u ; B_{k}^{i}\right) \leqslant \frac{1}{k}-\alpha \mu\left(F_{k}^{l_{\varepsilon}}\right) .
$$

By $\left(\mathrm{C}_{1}\right)$ and the locality condition $\left(\mathrm{C}_{3}\right)$ we have

$$
I\left(u+\varphi_{k, l_{\varepsilon}} ; F_{k}^{l_{\varepsilon}}\right)-I\left(u ; F_{k}^{l_{\varepsilon}}\right) \leqslant \frac{1}{k}-\alpha \mu\left(N_{\alpha}^{s}\right)+\varepsilon .
$$

since $\varphi_{k}^{i}=\varphi_{k, l_{\varepsilon}} \mu$-a.e. in $B_{k}^{i}$. But, by $\left(\mathrm{C}_{4}\right)$ we have $I\left(u+\varphi_{k, l_{\varepsilon}} ; B_{s} \backslash F_{k}^{l_{\varepsilon}}\right)-I\left(u ; B_{s} \backslash F_{k}^{l_{\varepsilon}}\right)=$ 0 and then

$$
I\left(u+\varphi_{k, l_{\varepsilon}} ; B_{s}\right)-I\left(u ; B_{s}\right)=I\left(u+\varphi_{k, l_{\varepsilon}} ; F_{k}^{l_{\varepsilon}}\right)-I\left(u ; F_{k}^{l_{\varepsilon}}\right) \leqslant \frac{1}{k}-\alpha \mu\left(N_{\alpha}^{s}\right)+\varepsilon .
$$

Letting $k \rightarrow \infty$ in $(7.6)$ and using the lower semicontinuity assumption $\left(\mathrm{C}_{u}\right)$ we have

$$
0 \leqslant \varliminf_{k \rightarrow \infty} I\left(u+\varphi_{k, l_{\varepsilon}} ; B_{s}\right)-I\left(u ; B_{s}\right) \leqslant-\alpha \mu\left(N_{\alpha}^{s}\right)+\varepsilon .
$$

Letting $\varepsilon \rightarrow 0$ we obtain $\mu\left(N_{\alpha}^{s}\right)=0$ which finishes the proof.

\section{REFERENCES}

[AF84] Emilio Acerbi and Nicola Fusco. Semicontinuity problems in the calculus of variations. Arch. Rational Mech. Anal., 86(2):125-145, 1984.

[AHM15] Omar Anza Hafsa and Jean-Philippe Mandallena. On the relaxation of variational integrals in metric Sobolev spaces. Adv. Calc. Var., 8(1):69-91, 2015.

[AHM17] Omar Anza Hafsa and Jean-Philippe Mandallena. $\Gamma$-convergence of nonconvex integrals in Cheeger-Sobolev spaces and homogenization. Adv. Calc. Var., 10(4):381-405, 2017.

[AHM18] Omar Anza Hafsa and Jean-Philippe Mandallena. Relaxation of nonconvex unbounded integrals with general growth conditions in Cheeger-Sobolev spaces. Bull. Sci. Math., 142:49-93, 2018.

[BB11] Anders Björn and Jana Björn. Nonlinear potential theory on metric spaces, volume 17 of EMS Tracts in Mathematics. European Mathematical Society (EMS), Zürich, 2011. 
[Bjö00] Jana Björn. $L^{q}$-differentials for weighted Sobolev spaces. Michigan Math. J., 47(1):151-161, 2000 .

[BM84] J. M. Ball and F. Murat. $W^{1, p}$-quasiconvexity and variational problems for multiple integrals. J. Funct. Anal., 58(3):225-253, 1984.

[Buc99] Stephen M. Buckley. Is the maximal function of a Lipschitz function continuous? Ann. Acad. Sci. Fenn. Math., 24(2):519-528, 1999.

[BZ90] J. M. Ball and K.-W. Zhang. Lower semicontinuity of multiple integrals and the biting lemma. Proc. Roy. Soc. Edinburgh Sect. A, 114(3-4):367-379, 1990.

[Che99] J. Cheeger. Differentiability of Lipschitz functions on metric measure spaces. Geom. Funct. Anal., 9(3):428-517, 1999.

[CM98] Tobias H. Colding and William P. Minicozzi, II. Liouville theorems for harmonic sections and applications. Comm. Pure Appl. Math., 51(2):113-138, 1998.

[CV77] C. Castaing and M. Valadier. Convex analysis and measurable multifunctions. Lecture Notes in Mathematics, Vol. 580. Springer-Verlag, Berlin-New York, 1977.

[Dac08] Bernard Dacorogna. Direct methods in the calculus of variations, volume 78 of Applied Mathematical Sciences. Springer, New York, second edition, 2008.

[FHK99] B. Franchi, P. Hajłasz, and P. Koskela. Definitions of Sobolev classes on metric spaces. Ann. Inst. Fourier (Grenoble), 49(6):1903-1924, 1999.

[Fra03] Ilaria Fragalà. Lower semicontinuity of multiple $\mu$-quasiconvex integrals. ESAIM Control Optim. Calc. Var., 9:105-124, 2003.

[GH13] Jasun Gong and Piotr Hajłasz. Differentiability of $p$-harmonic functions on metric measure spaces. Potential Anal., 38(1):79-93, 2013.

[GT01] Vladimir Gol'dshtein and Marc Troyanov. Axiomatic theory of Sobolev spaces. Expo. Math., 19(4):289-336, 2001.

[Haj03] Piotr Hajłasz. Sobolev spaces on metric-measure spaces. In Heat kernels and analysis on manifolds, graphs, and metric spaces (Paris, 2002), volume 338 of Contemp. Math., pages 173-218. Amer. Math. Soc., Providence, RI, 2003.

[Hei07] Juha Heinonen. Nonsmooth calculus. Bull. Amer. Math. Soc. (N.S.), 44(2):163-232, 2007.

[HK98] Juha Heinonen and Pekka Koskela. Quasiconformal maps in metric spaces with controlled geometry. Acta Math., 181(1):1-61, 1998.

[HKST15] Juha Heinonen, Pekka Koskela, Nageswari Shanmugalingam, and Jeremy T. Tyson. Sobolev spaces on metric measure spaces, volume 27 of New Mathematical Monographs. Cambridge University Press, Cambridge, 2015. An approach based on upper gradients.

[Kei04] Stephen Keith. A differentiable structure for metric measure spaces. Adv. Math., 183(2):271$315,2004$.

[Kri15] Jan Kristensen. A necessary and sufficient condition for lower semicontinuity. Nonlinear Anal., 120:43-56, 2015.

[Man05] Jean-Philippe Mandallena. Quasiconvexification of geometric integrals. Ann. Mat. Pura Appl. (4), 184(4):473-493, 2005.

[Man13] Jean-Philippe Mandallena. Lower semicontinuity via $W^{1, q}$-quasiconvexity. Bull. Sci. Math., 137(5):602-616, 2013.

[Mar85] Paolo Marcellini. Approximation of quasiconvex functions, and lower semicontinuity of multiple integrals. Manuscripta Math., 51(1-3):1-28, 1985.

[Mor52] Charles B. Morrey, Jr. Quasi-convexity and the lower semicontinuity of multiple integrals. Pacific J. Math., 2:25-53, 1952.

[Roc69] R.T Rockafellar. Measurable dependence of convex sets and functions on parameters. Journal of Mathematical Analysis and Applications, 28(1):4 - 25, 1969.

[Sha00] Nageswari Shanmugalingam. Newtonian spaces: an extension of Sobolev spaces to metric measure spaces. Rev. Mat. Iberoamericana, 16(2):243-279, 2000.

[Syc15] M. A. Sychev. Solution of a problem of Ball and Murat. Dokl. Akad. Nauk, 465(4):411-414, 2015 . 